Article

\title{
Protective Effects of Eicosapentaenoic Acid Plus Hydroxytyrosol Supplementation Against White Adipose Tissue Abnormalities in Mice Fed a High-Fat Diet
}

\author{
Paola Illesca ${ }^{1}\left(\mathbb{D}\right.$, Rodrigo Valenzuela ${ }^{2, *}{ }^{-}$, Alejandra Espinosa ${ }^{3}$, Francisca Echeverría ${ }^{2}{ }^{\infty}$, \\ Sandra Soto-Alarcon ${ }^{2}$, Cristian Campos ${ }^{3}$, Alicia Rodriguez ${ }^{4}\left(\mathbb{D}\right.$, Romina Vargas ${ }^{5}$, \\ Thea Magrone ${ }^{6}$ and Luis A. Videla ${ }^{5}$ (D) \\ 1 Laboratory of Studies of Metabolic Diseases Related to Nutrition. Faculty of Biochemistry. \\ University of Litoral, Santa Fe 3000, Argentina; pillesca@fbcb.unl.edu.ar \\ 2 Nutrition Department, Faculty of Medicine, University of Chile, Santiago 8380000, Chile; \\ franciscaecheverria@uchile.cl (F.E.); sandra.soto.alarcon@gmail.com (S.S.A.) \\ 3 Medical Technology Department, Faculty of Medicine, University of Chile, Santiago 8380000, Chile; \\ ealejand@uchile.cl (A.E.); cristian.campos.19@gmail.com (C.C.) \\ 4 Department of Food Science and Chemical Technology, Faculty of Chemical and Pharmaceutical Sciences, \\ University of Chile, Santiago 8380494, Chile; arodrigm@uchile.cl \\ 5 Molecular and Clinical Pharmacology Program, Institute of Biomedical Science, Faculty of Medicine, \\ University of Chile, Santiago 8380000, Chile; rominavargas@med.uchile.cl (R.V.); \\ lvidela1944@gmail.com (L.A.V.) \\ 6 Department of Basic Medical Sciences, Neuroscience and Sensory Organs, University of Bari, \\ 70124 Bari, Italy; thea.magrone@uniba.it \\ * Correspondence: rvalenzuelab@med.uchile.cl or hijodebello@gmail.com; Tel.: +56-2-29786014; \\ Fax: $+56-2-9786182$
}

Academic Editor: Filomena Conforti

Received: 27 August 2020; Accepted: 20 September 2020; Published: 27 September 2020

check for updates

\begin{abstract}
Objective: Obesity induced by high-fat diet (HFD) elicits white adipose tissue dysfunction In this study, we have hypothesized that the metabolic modulator eicosapentaenoic acid (EPA) combined with the antioxidant hydroxytyrosol (HT) attenuates HFD-induced white adipose tissue (WAT) alterations. Methods: C57BL/6J mice were administered with a HFD $(60 \%$ fat, $20 \%$ protein, $20 \%$ carbohydrates) or control diet (CD; $10 \%$ fat, $20 \%$ protein, $70 \%$ carbohydrates), with or without EPA $(50 \mathrm{mg} / \mathrm{kg} /$ day), HT ( $5 \mathrm{mg} / \mathrm{kg} /$ day), or both for 12 weeks. Determinations in WAT include morphological parameters, EPA and docosahexaenoic acid content in phospholipids (gas chromatography), lipogenesis, oxidative stress (OS) and inflammation markers, and gene expression and activities of transcription factors, such as sterol regulatory element-binding protein-1c (SREBP-1c), peroxisome proliferator-activated receptor-gamma (PPAR- $\gamma$ ), nuclear factor kappa-light-chain-enhancer of activated B cells (NF-kB) (p65 subunit) and nuclear factor erythroid 2-related factor 2 (Nrf2) (quantitative polymerase chain reaction and enzyme linked immunosorbent assay). Results: HFD led to WAT hypertrophy in relation to PPAR- $\gamma$ downregulation. WAT metabolic dysfunction was characterized by upregulation of lipogenic SREBP-1c system, mitochondrial energy metabolism depression, loss of the antioxidant Nrf2 signaling with OS enhancement, n-3 long-chain polyunsaturated fatty acids depletion and activation of the pro-inflammatory NF- $\mathrm{kB}$ system. EPA and HT co-supplementation diminished HFD-dependent effects additively, reaching values close or similar to controls. Conclusion: Data presented strengthen the importance of combined protocols such as EPA plus HT to attenuate metabolic-inflammatory states triggered by obesity.
\end{abstract}


Keywords: high-fat diet; white adipose tissue; adipocyte hypertrophy; metabolic dysfunction; eicosapentaenoic acid; hydroxytyrosol

\section{Introduction}

Overweight and obesity have been defined by the World Health Organization (WHO) as the abnormal or excessive fat accumulation that may impair health [1]. It currently represents a worrying public health problem, since from 1975-1980 to the present, the global prevalence of overweight and obesity has doubled, reaching a third of the world's population with this condition [2-4]. Obesity increases the risk of chronic noncommunicable diseases (NCDs) and triggers skeletal muscle disorders, sleep apnea and poor mental health [1], with development of non-alcoholic fatty liver disease (NAFLD) [5]. The fat accumulation that characterizes obesity promotes white adipose tissue (WAT) expansion, thus altering its normal functions. These include hypertrophy contributing to development of oxidative stress, the activation of low-grade inflammatory response and dysregulation of lipolysis accompanied by insulin resistance (IR) [6]. The prevention of obesity and associated syndromes has been assessed by the supplementation with different compounds, including natural products. Among them, the use of the n-3 long-chain polyunsaturated fatty acids (n-3 LCPUFAs), eicosapentaenoic acid (C20:5n-3, EPA) and docosahexaenoic acid (C22:6n-3, DHA) revealed effective outcomes not only related to lipid metabolism disorders, but also to cardiovascular diseases and inflammatory states [7]. EPA is biosynthesized from the essential precursor $\alpha$-linolenic acid ((C18:3n-3), ALA) through desaturation and elongation reactions occurring primarily in the liver, with actions being exerted on signal transduction pathways and gene transcription [8]. These include downregulation of lipogenic sterol regulatory element-binding protein-1c (SREBP-1c), upregulation of peroxisome proliferator-activated receptor- $\alpha$ (PPAR- $\alpha$ ) favoring fatty acid oxidation (FAO) and promotion of inflammation resolution via synthesis of specific pro-resolving mediators (SPMs) [9]. Besides $n-3$ LCPUFAs, antioxidant compounds also have positive results in NCDs, as is the case of the polyphenol hydroxytyrosol (HT) present in olive oil and red wine. HT has biological properties beyond its potent antioxidant capacity inhibiting oxidative stress [10], such as the modulation of lipid metabolism and inflammatory responses [11]. Both EPA and HT can activate transcription factors that modify the gene expression of downstream lipid, antioxidant and inflammatory mediators [11-13].

Currently, preclinical studies point to the advantage of using combined protocols over monotherapies in the prevention or resolution of metabolic disorders [14,15]. In fact, the co-supplementation protocols are more effective than the single ones, considering that the former exhibit the possibility to exert additive or potentiated responses [14,16]. In this respect, high-fat diet (HFD)-induced liver steatosis and pro-inflammatory status in mice were attenuated by combined EPA and HT administration in an additive manner $[17,18]$. The underlying mechanisms comprise the regulation of SREBP-1c and PPAR- $\alpha$ controlling lipogenesis and FAO respectively, as well as the content of the SPMs resolvin-1 and 2 abrogating inflammation [17,18]. According to these considerations, the aim of this study was to test the hypothesis that the co-administration of EPA and HT potentiates the effects of the separate supplementation with EPA or HT on adiposity enhancement and WAT dysfunction in HFD-fed mice.

\section{Material and Methods}

\subsection{Animals, Diets and Experimental Procedures}

All animal procedures in this study were in strict adherence to the Guide for the Care and Use of Laboratory Animals (National Academy of Sciences, NIH Publication 6-23, revised 1985) and were approved by the Bioethics Committee for Research in Animals, Faculty of Medicine, University of Chile (CBA \# 0580 FMUCH). Weaning male C57BL/6J mice weighing 12-14 g (Bioterio Central, ICBM, Faculty of Medicine, University of Chile) were randomly assigned to each experimental group and 
allowed free access to a control diet (CD) or HFD. The composition of CD (expressed as \% total calories) was $10 \%$ fat, $20 \%$ protein and $70 \%$ carbohydrate, with a caloric value of $3.85 \mathrm{kcal} / \mathrm{g}$ and free of EPA and DHA. The composition of the HFD was $60 \%$ fat, $20 \%$ protein and $20 \%$ carbohydrate, with a caloric value of $5.24 \mathrm{kcal} / \mathrm{g}$ and free of EPA and DHA (Research Diet INC, Rodent Diet, Product data D12450K and 12492, USA). Fatty acid composition of CD and HFD were previously published [19]. Animals received water ad libitum and were housed on a $12 \mathrm{~h}$ light/dark cycle from day 1 to 84 (12 weeks). As appropriate, the treated groups received orally through gavage, EPA and/or HT. EPA, isolated from fish oil (Golden Omega S.A., Chile) as triacylglycerols (TAG) (50\% EPA, 5\% DHA and 5\% of other n-3 FAs, 15\% saturated fatty acid (SFA) principally palmitic acid and 25\% monounsaturated fatty acids (MUFAs) principally oleic acid). EPA was administered at $50 \mathrm{mg} / \mathrm{kg} /$ day dosage. HT (ela-Vida ${ }^{\mathrm{TM}}$, DSM Nutritional Products Company, Netherlands) was given at doses of $5 \mathrm{mg} / \mathrm{kg} /$ day. Control groups received isovolumetric amounts of saline orally, conforming eight experimental groups, namely: (a) CD + saline (control), (b) CD + EPA, (c) CD + HT, (d) CD + EPA + HT, (e) HFD + saline, (f) HFD + EPA, (g) HFD + HT and (h) HFD + EPA + HT. The doses of EPA and HT used in this study were in agreement with previous investigations: EPA (50 mg/ $\mathrm{kg}$ ) and HT (5 mg/kg), both supplied separately or in combination, and showed protective effects against HFD-induced steatosis [20]. In addition, HT at the doses mentioned above proved to prevent adipose tissue dysfunction induced by HFD [14]. Weekly controls of body weight (BW) and diet intake were performed through the whole period. At the end of the 12th week, animals were fasted (6-8 h). Under isoflurane anesthesia, subcutaneous, perirenal and epididymal fat pads were removed, weighed and immediately assayed or frozen in liquid nitrogen as appropriate. Epididymal white adipose tissue (EWAT) dissection was performed carefully to avoid contamination with subcutaneous adipose tissue. Blood samples were collected, and serum was obtained after centrifugation $\left(1000 \times g, 10 \mathrm{~min}, 4^{\circ} \mathrm{C}\right)$ for later analysis of adipokines. The animals were euthanized by cardiac puncture. EWAT was selected to perform the evaluations since it is metabolically representative of visceral adipose tissue in rodents and is the largest and most accessible fat pad.

\subsection{Serum Adipokines Profile}

ELISA kits were used for the assessment of serum levels of adiponectin (mg/L) (Cayman Chemical Company, number 10007620, Ann Arbor, MI, USA) and leptin (mg/mL) (Abcam Inc., number ab100718, Toronto, ON, Canada).

\subsection{Morphological Parameters}

Adipocytes from EWAT were isolated according to the method of Rodbell [21], as previously described [13]. The morphological parameters of adipocytes (size, mean diameter and cell volume) were determined according to Di Girolamo et al. [22]. The number of cells in the fat pad was estimated by measuring the lipid content by the method of Folch [23]. The lipid content of the average fat cell and the total number of cells in the fat pad was calculated as recently described in detail [13].

\subsection{Fatty Acid Composition of Phospholipids}

The methodology used to assess phospholipid fatty acid composition was described elsewhere $[13,24]$. Briefly, the total lipids from EWAT were extracted with chloroform/methanol $(2: 1 v / v)$ according to the method proposed by Bligh and Dyer [25]. Then, the phospholipids were isolated from the lipid extract by thin-layer chromatography performed on silica gel plates. The derivatization of phospholipids was carried out with boron trifluoride to produce fatty acid methyl esters (FAME), which were subsequently separated and quantified by gas-liquid chromatography.

\subsection{Oxidative Stress Indicators and Non-Enzymatic Defenses}

EWAT thiobarbituric acid reactive substances (TBARS) and F-2 isoprostanes contents were determined by colorimetric assays according to the manufacturer's instructions (Cayman Chemical Company, Ann Arbor, MI, USA). Protein carbonyls concentration was determined by a fluorometric 
assay (Cayman Chemical Company, Ann Arbor, MI, USA) after adjusting the total protein concentration to $7.5 \mathrm{mg} / \mathrm{mL}$ per sample. Reduced glutathione (GSH) and glutathione disulfide (GSSG) contents in EWAT were assessed with an enzymatic recycling method described by Rahman et al. [26].

\subsection{Activities of Antioxidant and Lipogenic Enzymes}

Samples of EWAT were homogenized in three volumes of phosphate buffer (30 mM), pH 7.4 containing EDTA $(1 \mathrm{mM})$ and sucrose $(250 \mathrm{mM})$ for determination of antioxidant enzyme activities. The homogenate was subjected to a first centrifugation $\left(1200 \times \mathrm{g}, 10 \mathrm{~min}, 4^{\circ} \mathrm{C}\right)$ and one supernatant aliquot was used to evaluate superoxide dismutase (SOD) (Cayman Chemical Co., Ann Arbor, MI, USA) and catalase (CAT) activities [27]. Subsequently, the remaining supernatants were centrifuged at $100,000 \times \mathrm{g}$ for $60 \mathrm{~min}$ at $4{ }^{\circ} \mathrm{C}$ to perform glutathione peroxidase (GPX) and glutathione reductase (GR) assays [27]. On the other hand, EWAT samples were homogenized in three volumes of buffer, $\mathrm{pH} 7$, containing $\mathrm{KH}_{2} \mathrm{PO}_{4}(9 \mathrm{mM}), \mathrm{K}_{2} \mathrm{HPO}_{4}(85 \mathrm{mM})$, dithiothreitol (DTT) $(1 \mathrm{mM})$ and $\mathrm{KHCO}_{3}$ $(70 \mathrm{mM})$ and centrifuged $\left(1800 \times \mathrm{g}, 10 \mathrm{~min}, 4{ }^{\circ} \mathrm{C}\right)$. The fat of the upper layers were discarded, the supernatants were again centrifuged at $100,000 \times \mathrm{g}$ for $60 \mathrm{~min}$ at $4{ }^{\circ} \mathrm{C}$ and the aqueous fractions were used to study Acetyl-CoA carboxylase (ACC), fatty acid synthase (FAS) and the glucose-6-phosphate dehydrogenase (G6PD) activities as previously described [12,14]. To assay malic enzyme (ME) activity, EWAT homogenates were centrifuged for $10 \mathrm{~min}$ at $40,000 \times \mathrm{g}$ at $4{ }^{\circ} \mathrm{C}$, then the ME activity was evaluated in the resulting supernatant following the method performed by Wise and Ball [28]. In addition, the activity of lipoprotein lipase (LPL) (enzyme associated with the tissue incorporation of fatty acids) was measured in an epididymal fat pad according to Llobera et al. [29]. The protein content in enzyme extracts was determined by the Bradford assay (Bio-Rad reagent, California, USA).

\subsection{SREBP-1c, PPAR- $\gamma, N F-K B$ and Nrf2 DNA-Binding Activity}

Nuclear extracts from EWAT were obtained using a commercial extraction kit (Cayman Chemical Company number 78782-99-7, Ann Arbor, MI, USA). SREBP-1c, PPAR- $\gamma$, nuclear factor-kB (NF- $k B$ ) (p65 subunit) and nuclear factor E2-related factor 2 (Nrf2) DNA-binding activities were assessed with a commercial ELISA kit (Cayman Chemical Company, number 10010854. SREBP-1c, number 10006855 by PPAR- $\gamma$, number 10007889 by NF-kB (p65) and number 600590 by Nrf2, Ann Arbor, MI, USA) and according to the manufacturer's instructions. Values were expressed as percentage of SREBP-1c, PPAR- $\gamma$, NF- $\mathrm{kB}$ (p65 subunit) and Nrf2 DNA-binding with respect to a positive control provided by the respective ELISA kit.

\subsection{Quantitative Real-Time PCR}

Total RNA was isolated from EWAT samples using Trizol (Invitrogen, Carlsbad, CA, USA), according to the supplier's protocols. Purified RNA $(2 \mu \mathrm{g})$ was then treated with DNAase I (DNA free kit; Ambion, Austin, TX, USA) and used to generate first-strand cDNA with moloney murine leukemia virus reverse transcriptase (Invitrogen, Carlsbad, CA, USA), utilizing random hexamers (Invitrogen, Carlsbad, CA, USA) and deoxynucleotide triphosphate (dNTP) mix (Bioline, London, United Kingdom), according to the manufacturer's protocol. The resultant cDNA was amplified with specific primers for mice in a total volume of $20 \mu \mathrm{L}$. Gene-specific primer sequences used are shown in Table 1. Primers were optimized to yield $95 \%-100 \%$ of reaction efficiency with PCR products by development in agarose gel to verify the correct amplification length. The quality of the DNA extracted was checked by agarose gel (1\%) electrophoresis according to Lee et al. [30]. Briefly, samples were mixed with Gel loading dye $(0.25 \%$ bromophenol blue, $0.25 \%$ xylene cyanol and 30\% glycerol). Electrophoresis was run at 75 Volt for 20 min. DNA was stained using GelRed ${ }^{\circledR}$ Nucleic Acid Gel Stain (Biotum, Fremont, CA, USA). Real-Time PCR was performed in a Stratagen Mx3000P System using Brilliant II SYBR ${ }^{\circledR}$ Green quantitative real time PCR Master Mix (Agilent Technologies, La Jolla, CA, USA) following the manufacturer's recommendations (Applied Biosystems, Foster City, CA, USA). All the expression levels of target genes under study were normalized by the expression of $\beta$-actin as 
an internal control (Applied Biosystems, Foster City, CA, USA). Fold changes between groups were calculated by the 2-( $\Delta \Delta \mathrm{Ct})$ method, as established by Pfaffl [31] and Livak and Schmittgen [32]. 
Table 1. Gene-specific primer sequences used in the study.

\begin{tabular}{|c|c|c|c|c|c|}
\hline mRNA & Forward Primer & Reverse Primer & Melting $\mathrm{T}^{\mathrm{o}}$ & Product Length & Gen Bank Code \\
\hline Nrf2 & AAGCTTTCAACCCGAAGCAC & TTTCCGAGTCACTGAACCCA & $58^{\circ} \mathrm{C}$ & 156 & NM_0317899.2 \\
\hline GST & TGCAGACCAAAGCCATTCTC & ACGGTTCCTGGTTTGTTCCT & $56^{\circ} \mathrm{C}$ & 197 & NM_017013.4 \\
\hline GCL & ATGTGGACACCCGATGCAGTATT & TGTCTTGCTTGTAGTCAGGATGGTTT & $62{ }^{\circ} \mathrm{C}$ & 129 & NM_012815.2 \\
\hline SREBP-1c & CTGGAGACATCGCAAACAAGC & ATGGTAGACAACAGCCGCATC & $58^{\circ} \mathrm{C}$ & 277 & NM_001358315.1 \\
\hline FAS & ATCCTGGAACGAGAACACGATCA & AGAGACGTGTCACTCCTGGACTT & $59^{\circ} \mathrm{C}$ & 140 & NM_017332.1 \\
\hline $\mathrm{ACC}$ & ACCAGGGCAAATGCATCAGT & TCGGAAAAGCATCGGGAAGT & $57^{\circ} \mathrm{C}$ & 185 & NM_022193.1 \\
\hline PPAR- $\gamma$ & CCAGAGCATGGTGCCTTCGCT & CAGCAACCATTGGGTCAGCTC & $58^{\circ} \mathrm{C}$ & 241 & NM_011146.3 \\
\hline FATP1 & TCCGTCTGGTCAAGGTCAAT & GAAAACGCTGTGGGCAATCT & $56^{\circ} \mathrm{C}$ & 188 & NM_011977.4 \\
\hline LPL & AGCCAGGATGCAACATTGGA & TTGCACCTGTATGCCTTGCT & $57^{\circ} \mathrm{C}$ & 157 & NM_008509.2 \\
\hline$N F-k B$ & GAGGTCTCTGGGGGTACCAT & AAGGCTGCCTGGATCACTTC & $58^{\circ} \mathrm{C}$ & 89 & NM_008689.2 \\
\hline TNF- $\alpha$ & ATGGCCTCCСТCTCATCAGT & TTTGCTACGACGTGGGCTAC & $58^{\circ} \mathrm{C}$ & 97 & NM_013693.3 \\
\hline IL-6 & TCCATCCAGTTGCCTTCTTG & TTCCACGATTTCCCAGAGAAC & $56^{\circ} \mathrm{C}$ & 167 & NM_031168.2 \\
\hline$\beta$-actin & ACTGCCGCATCCTCTTCCTC & CTCCTGCTTGCTGATCCACATC & $58^{\circ} \mathrm{C}$ & 399 & NM_031144.3 \\
\hline
\end{tabular}

( (PAR- $\gamma)$, Fatty acid transport protein 1 (FATP1), lipoprotein lipase (LPL), nuclear factor kappa-light-chain-enhancer of activated B cells (NF-KB), tumor necrosis factor alpha (TNF- $\alpha$ ), interleukin 6 (IL-6), beta actin ( $\beta$-actin). 


\subsection{Statistical Analysis}

Statistical analysis was performed with GraphPad Prism 6.0 software (GraphPad Prism, Inc., San Diego, USA). The values shown represent the mean \pm standard deviation (SD) for 8-10 animals per each experimental group. Evaluation of normality data distribution was performed using the Shapiro-Wilk test. Assessment of the statistical significance of differences between mean values was performed by two-way analysis of variance (ANOVA) and the Tukey's test. A $p<0.05$ was considered significant.

\section{Results}

\subsection{HFD-Induced Obesity, Adiposity and WAT Hypertrophy are Decreased by EPA and HT Co-Administration}

The body weight (BW) of mice at the beginning of the study was similar. After 12 weeks, all groups fed with CD, with or without supplementation, exhibited comparable final BW. HFD led to an increase in BW gain of $65 \%(p<0.05)$, compared with CD groups, which is related with to augmented adiposity (Table 2A, group e). In fact, all fat pads evaluated, namely, subcutaneous, perirenal and epididymal, in mice fed the HFD showed significant weight increases (Table 2B), therefore the total mass of WAT increased considerably $(198 \% ; p<0.05)$ over the CD group. Dietary intake was comparable in all experimental groups, but energy consumption was higher in mice subjected to the HFD with or without supplementation over CD groups (Data not shown). EPA and EPA + HT supplementation diminished BW gain produced by the HFD by $30 \%$ and $37 \%$ respectively $(p<0.05)$, accompanied with a decrease in fat pads and total WAT of $34 \%$ and $32 \%(p<0.05)$, respectively (Table $2 \mathrm{~B})$. HT administration was not able to prevent the increased BW observed in HDF-fed mice; however, it reduced the localized and total adiposity (total WAT) by $28 \%$ (Table 2B). Specific and morphological parameters were evaluated in the EWAT (Table 2C). The expansion of epididymal fat pad of HFD-fed mice exhibited a lower number of cells per gram of WAT, adipocytes of increased size with augmented TAG content compared with the control group, indicating hypertrophy. The supplementation with EPA, HT or both significantly decreased the hypertrophy observed in WAT of the HFD-fed mice, improving all the parameters evaluated without observing differences between treatments and without reaching control values (Table 2C). Levels of serum adipokines are shown in the Table 2D. Serum adiponectin and leptin were not modified by supplementation when mice were fed the CD. However, the HFD significantly decreased serum adiponectin levels $(39 \% ; p<0.05)$ while increasing those of leptin $(136 \% ; p<0.05)$. Supplementation with EPA, HT or EPA + HT improved the profile of serum adipokines in HFD-fed mice, showing a similar effect independently of the treatment, although the values did not reach those observed in CD-fed mice.

\subsection{HFD-Induced Changes in Fatty Acid Composition in EWAT Phospholipids are Improved by EPA and HT Co-Supplementation}

Comparable content of total saturated fatty acids (SFA), monounsaturated fatty acids (MUFA) and polyunsaturated fatty acids (PUFA) were found in EWAT phospholipids of all groups receiving CD. HFD-fed mice exhibit an increase of total SFA and decrease of total PUFA (Table 3). Regarding the EWAT palmitate levels, comparable values were observed in all groups fed with $\mathrm{CD}$, a significant increment in HFD saline group, and a decrease caused by the administration of EPA or HT alone, with the total recovery towards CD values when supplemented with EPA+HT (Table 3). Moreover, HFD led to a severe reduction in EPA content $(81 \% ; p<0.05)$ with consequent decrease in DHA content $(72 \%$; $p<0.05$ ) in EWAT phospholipids compared to CD-fed animals (Table 3). The high content of EPA in the adipose tissue of the animals that received the supplementation evidence the effectiveness of the treatment, increasing by 2.86-fold and 3-fold EPA levels in CD groups supplemented with $\mathrm{EPA}$ and EPA+HT, respectively. Moreover, the supplementation with EPA to HFD-mice (EPA and $\mathrm{EPA}+\mathrm{HT}$ groups) restored the depleted levels of EPA in EWAT phospholipids reaching control levels. The incorporation of EPA into EWAT also improved the content of DHA in this tissue, increasing DHA 
levels in CD animals, with normalization in HFD mice achieving levels observed in CD group (Table 3). It should be noted that in the HFD-fed group that received HT exclusively, the levels of EPA and DHA were also increased in EWAT phospholipids, confirming previous results $[18,20]$. Surprisingly, the effect of the supplements given together, improved the tissue content of EPA and DHA even more than when each administered individually in animals fed HFD. Furthermore, EPA reduced the n-6/n-3 ratio EWAT of CD animals, and ameliorate this ratio in both groups of mice fed HFD that received EPA supplementation. Also, HT given alone diminished the ratio n-6/n-3 in EWAT of HFD-mice, an effect that was more pronounce after EPA and HT co-supplementation (Table 3). 
Table 2. General and metabolic parameters in control diet (CD) and high-fat diet (HFD)-fed mice subjected to eicosapentaenoic acid (EPA), hydroxytyrosol (HT) or EPA + HT supplementation.

\begin{tabular}{|c|c|c|c|c|c|c|c|c|}
\hline & \multicolumn{8}{|c|}{ Groups } \\
\hline & \multicolumn{4}{|c|}{ Control Diet (CD) } & \multicolumn{4}{|c|}{ High-Fat Diet (HFD) } \\
\hline & Saline (a) & EPA (b) & HT (c) & EPA + HT (d) & Saline (e) & EPA (f) & HT (g) & EPA + HT (h) \\
\hline \multicolumn{9}{|l|}{ A. General parameters } \\
\hline Initial body weight (g) & $14.2 \pm 1.1$ & $13.9 \pm 1.4$ & $14.0 \pm 1.5$ & $14.3 \pm 1.1$ & $14.2 \pm 1.3$ & $13.8 \pm 1.3$ & $14.3 \pm 1.5$ & $13.9 \pm 1.3$ \\
\hline Final body weight $(\mathrm{g})$ & $37.2 \pm 3.1^{\mathrm{e}, \mathrm{f}, \mathrm{g}, \mathrm{h}}$ & $36.9 \pm 3.2^{e, f, g, h}$ & $39.6 \pm 3.5^{\mathrm{e}, \mathrm{f}, \mathrm{g}, \mathrm{h}}$ & $37.4 \pm 3.4^{\mathrm{e}, \mathrm{f}, \mathrm{g}, \mathrm{h}}$ & $53.2 \pm 4.4^{\mathrm{a}, \mathrm{b}, \mathrm{c}, \mathrm{d}, \mathrm{f}, \mathrm{h}}$ & $45.9 \pm 4.2^{\mathrm{a}, \mathrm{b}, \mathrm{c}, \mathrm{d}, \mathrm{e}}$ & $47.1 \pm 4.0^{\mathrm{a}, \mathrm{b}, \mathrm{c}, \mathrm{d}}$ & $44.3 \pm 3.6^{\mathrm{a}, \mathrm{b}, \mathrm{c}, \mathrm{d}, \mathrm{e}}$ \\
\hline \multicolumn{9}{|l|}{$\begin{array}{l}\text { B. White Adipose } \\
\text { Tissue (WAT) }\end{array}$} \\
\hline Subcutaneous WAT (g) & $0.27 \pm 0.09 \mathrm{e}, \mathrm{f}, \mathrm{g}, \mathrm{h}$ & $0.26 \pm 0.10^{\mathrm{e}, \mathrm{f}, \mathrm{g}, \mathrm{h}}$ & $0.28 \pm 0.11^{\mathrm{e}, \mathrm{f}, \mathrm{g}, \mathrm{h}}$ & $0.26 \pm 0.08^{e, f, g, h}$ & $0.88 \pm 0.17 \mathrm{a}, \mathrm{b}, \mathrm{c}, \mathrm{d}, \mathrm{f}, \mathrm{g}, \mathrm{h}$ & $0.51 \pm 0.15^{\mathrm{a}, \mathrm{b}, \mathrm{c}, \mathrm{d}, \mathrm{e}}$ & $0.57 \pm 0.13^{\mathrm{a}, \mathrm{b}, \mathrm{c}, \mathrm{d}, \mathrm{e}}$ & $0.49 \pm 0.17 \mathrm{a}, \mathrm{b}, \mathrm{c}, \mathrm{d}, \mathrm{e}$ \\
\hline Perirenal WAT (g) & $0.25 \pm 0.11 \mathrm{e}^{\mathrm{e}, \mathrm{f}, \mathrm{g}, \mathrm{h}}$ & $0.23 \pm 0.09$ e,f,h, $g$ & $0.27 \pm 0.12$ e,f,g,h & $0.22 \pm 0.07$ e,f,g,h & $0.67 \pm 0.19 a, b, c, d$ & $0.49 \pm 0.16^{a, b, c, d}$ & $0.59 \pm 0.16^{\mathrm{a}, \mathrm{b}, \mathrm{c}, \mathrm{d}}$ & $0.44 \pm 0.13^{\mathrm{a}, \mathrm{b}, \mathrm{b}, \mathrm{d}, \mathrm{d}, \mathrm{e}}$ \\
\hline Epididymal WAT (g) & $0.53 \pm 0.15^{\mathrm{e}, \mathrm{f}, \mathrm{g}, \mathrm{h}}$ & $0.51 \pm 0.11^{\mathrm{e}, \mathrm{f}, \mathrm{g}, \mathrm{h}}$ & $0.55 \pm 0.13^{\mathrm{e}, \mathrm{f}, \mathrm{g}, \mathrm{h}}$ & $0.50 \pm 0.11^{\mathrm{e}, \mathrm{f}, \mathrm{g}, \mathrm{h}}$ & $1.74 \pm 0.25^{\mathrm{a}, \mathrm{b}, \mathrm{c}, \mathrm{d}, \mathrm{f}, \mathrm{g}, \mathrm{h}}$ & $1.15 \pm 0.22 \mathrm{a}, \mathrm{b}, \mathrm{c}, \mathrm{d}, \mathrm{e}$ & $1.21 \pm 0.19 \mathrm{a}, \mathrm{b}, \mathrm{c}, \mathrm{d}, \mathrm{e}$ & $1.17 \pm 0.16^{\mathrm{a}, \mathrm{b}, \mathrm{c}, \mathrm{d}, \mathrm{e}}$ \\
\hline Total weigth WAT (g) & $1.12 \pm 0.12^{\mathrm{e}, \mathrm{f}, \mathrm{g}, \mathrm{h}}$ & $1.09 \pm 0.14^{\mathrm{e}, \mathrm{f}, \mathrm{g}, \mathrm{h}}$ & $1.15 \pm 0.14^{\mathrm{e}, \mathrm{f}, \mathrm{g}, \mathrm{h}}$ & $1.06 \pm 0.12^{\mathrm{e}, \mathrm{f}, \mathrm{g}, \mathrm{h}}$ & $3.34 \pm 0.31 \mathrm{a}, \mathrm{b}, \mathrm{c}, \mathrm{d}, \mathrm{f}, \mathrm{g}, \mathrm{h}$ & $2.21 \pm 0.20^{a, b, c, d, e}$ & $2.40 \pm 0.25^{\mathrm{a}, \mathrm{b}, \mathrm{c}, \mathrm{d}, \mathrm{e}}$ & $2.26 \pm 0.22^{a, b, c, d, e}$ \\
\hline \multicolumn{9}{|l|}{$\begin{array}{l}\text { C. Specific parameter of } \\
\text { Epididymal WAT }\end{array}$} \\
\hline $\begin{array}{l}\text { Relative weight }(\mathrm{g} / 100 \mathrm{~g} \\
\text { body weight) }\end{array}$ & $1.67 \pm 0.16^{\mathrm{e}, \mathrm{f}, \mathrm{g}, \mathrm{h}}$ & $1.65 \pm 0.13$ e,f,g,h & $1.63 \pm 0.16^{\mathrm{e}, \mathrm{f}, \mathrm{g}, \mathrm{h}}$ & $1.62 \pm 0.14^{\mathrm{e}, \mathrm{f}, \mathrm{g}, \mathrm{h}}$ & 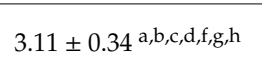 & $2.09 \pm 0.20^{\mathrm{a}, \mathrm{b}, \mathrm{c}, \mathrm{d}, \mathrm{e}}$ & $2.15 \pm 0.23^{\mathrm{a}, \mathrm{b}, \mathrm{c}, \mathrm{d}, \mathrm{e}}$ & $2.05 \pm 0.21^{\mathrm{a}, \mathrm{b}, \mathrm{c}, \mathrm{d}, \mathrm{e}}$ \\
\hline Cell volumen $(\mathrm{pL})$ & $259.4 \pm 22.0^{e, f, g, h}$ & $240.4 \pm 20.1^{\mathrm{e}, \mathrm{f}, \mathrm{g}, \mathrm{h}}$ & $248.5 \pm 23.3^{\mathrm{e}, \mathrm{f}, \mathrm{g}, \mathrm{h}}$ & $239.4 \pm 24.2^{e, f, g, h}$ & $498 \pm 40.2^{\mathrm{a}, \mathrm{b}, \mathrm{c}, \mathrm{d}, \mathrm{f}, \mathrm{g}, \mathrm{h}}$ & $348.4 \pm 30.3^{\mathrm{a}, \mathrm{b}, \mathrm{c}, \mathrm{d}, \mathrm{e}}$ & $351.1 \pm 40.6^{\mathrm{a}, \mathrm{b}, \mathrm{c}, \mathrm{d}, \mathrm{e}}$ & $342.1 \pm 27.7^{\mathrm{a}, \mathrm{b}, \mathrm{c}, \mathrm{d}, \mathrm{e}}$ \\
\hline $\begin{array}{l}\text { Cell number } \\
\left(\times 10^{6} / \mathrm{g} \text { tissue }\right)\end{array}$ & $4.15 \pm 0.37^{\mathrm{e}, \mathrm{f}, \mathrm{g}, \mathrm{h}}$ & $4.11 \pm 0.29 \mathrm{e}, \mathrm{f}, \mathrm{g}, \mathrm{h}$ & $4.17 \pm 0.32 \mathrm{e}, \mathrm{f}, \mathrm{g}, \mathrm{h}$ & $4.09 \pm 0.25^{\mathrm{e}, \mathrm{f}, \mathrm{g}, \mathrm{h}}$ & $2.91 \pm 0.21$ a,b,c,d,f,g,, $\mathrm{h}$ & $3.32 \pm 0.28^{\mathrm{a}, \mathrm{b}, \mathrm{c}, \mathrm{d}, \mathrm{e}}$ & $3.26 \pm 0.21^{\mathrm{a}, \mathrm{b}, \mathrm{c}, \mathrm{d}, \mathrm{e}}$ & $3.40 \pm 0.33^{a, b, c, d, e}$ \\
\hline $\begin{array}{c}\text { Triacylglicerols } \\
\text { (nmol/cell) }\end{array}$ & $0.37 \pm 0.099^{\mathrm{e}, \mathrm{f}, \mathrm{g}, \mathrm{h}}$ & $0.35 \pm 0.10^{\mathrm{e}, \mathrm{f}, \mathrm{g}, \mathrm{h}}$ & $0.34 \pm 0.11^{\mathrm{e}, \mathrm{f}, \mathrm{g}, \mathrm{h}}$ & $0.31 \pm 0.08^{\mathrm{e}, \mathrm{f}, \mathrm{g}, \mathrm{h}}$ & $0.68 \pm 0.18^{a, b, c, d, f, g, h}$ & $0.45 \pm 0.10^{a, b, c, d, e}$ & $0.49 \pm 0.13^{a, b, c, d, e}$ & $0.40 \pm 0.07^{\mathrm{a}, \mathrm{b}, \mathrm{c}, \mathrm{d}, \mathrm{e}}$ \\
\hline \multicolumn{9}{|l|}{ D. Serum parameters } \\
\hline Adiponectin (mg/L) & $3.55 \pm 0.21^{\mathrm{e}, \mathrm{f}, \mathrm{g}, \mathrm{h}}$ & $3.59 \pm 0.22 \mathrm{e}, \mathrm{f}, \mathrm{g}, \mathrm{h}$ & $3.48 \pm 0.24^{\mathrm{e}, \mathrm{f}, \mathrm{g}, \mathrm{h}}$ & $3.50 \pm 0.24^{\mathrm{e}, \mathrm{f}, \mathrm{g}, \mathrm{h}}$ & $2.15 \pm 0.14^{\mathrm{a}, \mathrm{b}, \mathrm{c}, \mathrm{d}, \mathrm{f}, \mathrm{g}, \mathrm{g}, \mathrm{h}}$ & $3.02 \pm 0.14^{\mathrm{a}, \mathrm{b}, \mathrm{c}, \mathrm{d}, \mathrm{e}}$ & $3.06 \pm 0.17 \mathrm{a}, \mathrm{b}, \mathrm{c}, \mathrm{d}, \mathrm{e}$ & $3.12 \pm 0.15^{\mathrm{a}, \mathrm{b}, \mathrm{c}, \mathrm{d}, \mathrm{e}}$ \\
\hline Leptin $(\mathrm{mg} / \mathrm{mL})$ & $1.14 \pm 0.08^{\mathrm{e}, \mathrm{f}, \mathrm{g}, \mathrm{h}}$ & $1.09 \pm 0.10^{\mathrm{e}, \mathrm{f}, \mathrm{g}, \mathrm{h}}$ & $1.15 \pm 0.11^{\mathrm{e}, \mathrm{f}, \mathrm{g}, \mathrm{h}}$ & $1.10 \pm 0.08^{\text {eff,g.h }}$ & $2.69 \pm 0.29^{a, b, c, d, f, g, h}$ & $1.83 \pm 0.20^{\mathrm{a}, \mathrm{b}, \mathrm{c}, \mathrm{d}, \mathrm{e}}$ & $1.91 \pm 0.26^{\mathrm{a}, \mathrm{b}, \mathrm{c}, \mathrm{d}, \mathrm{e}}$ & $1.78 \pm 0.17 \mathrm{a}, \mathrm{b}, \mathrm{b}, \mathrm{c}, \mathrm{d}, \mathrm{e}$ \\
\hline
\end{tabular}


Table 3. Most relevant fatty acid composition of epididymal white adipose tissue (WAT) phospholipids obtained from control diet- (CD) and high-fat diet (HFD)-fed mice subjected to eicosapentaenoic acid (EPA), hydroxytyrosol (HT) or EPA plus HT supplementation.

\begin{tabular}{|c|c|c|c|c|c|c|c|c|}
\hline \multirow[b]{3}{*}{ Fatty Acid } & \multicolumn{8}{|c|}{ Fatty Acid Composition (g/100 g FAME) } \\
\hline & \multicolumn{4}{|c|}{ Control Diet (CD) } & \multicolumn{4}{|c|}{ High-Fat Diet (HFD) } \\
\hline & Saline (a) & EPA (b) & HT (c) & $\mathrm{EPA}+\mathrm{HT}(\mathrm{d})$ & Saline (e) & EPA (f) & HT (g) & $\mathrm{EPA}+\mathrm{HT}(\mathrm{h})$ \\
\hline $\mathrm{C} 16: 0$ & $36.5 \pm 3.9^{\mathrm{e}, \mathrm{f}, \mathrm{g}}$ & $33.7 \pm 4.5^{\mathrm{e}, \mathrm{f}, \mathrm{g}, \mathrm{h}}$ & $34.9 \pm 3.7^{\mathrm{e}, \mathrm{f}, \mathrm{g}, \mathrm{h}}$ & $34.8 \pm 3.2^{\mathrm{e}, \mathrm{f}, \mathrm{g}, \mathrm{h}}$ & $48.7 \pm 4.9^{\mathrm{a}, \mathrm{b}, \mathrm{c}, \mathrm{d}, \mathrm{f}, \mathrm{g}, \mathrm{g}, \mathrm{h}}$ & $40.8 \pm 4.1^{\mathrm{a}, \mathrm{b}, \mathrm{c}, \mathrm{d}, \mathrm{e}}$ & $41.5 \pm 4.6^{\mathrm{a}, \mathrm{b}, \mathrm{c}, \mathrm{d}, \mathrm{e}}$ & $39.8 \pm 3.5^{\mathrm{b}, \mathrm{c}, \mathrm{d}, \mathrm{e}}$ \\
\hline $\mathrm{C} 18: 0$ & $8.04 \pm 1.2$ & $8.12 \pm 1.4$ & $8.19 \pm 1.5$ & $8.16 \pm 1.2$ & $8.05 \pm 1.8$ & $8.11 \pm 1.5$ & $8.54 \pm 1.3$ & $8.14 \pm 1.4$ \\
\hline C18:1n-9 & $27.2 \pm 2.8$ & $26.4 \pm 3.0$ & $27.1 \pm 3.1$ & $27.4 \pm 2.5$ & $25.2 \pm 3.2$ & $27.1 \pm 2.1$ & $23.5 \pm 2.4$ & $25.9 \pm 2.8$ \\
\hline C18:2n-6 (LA) & $8.12 \pm 1.3$ & $8.22 \pm 1.2$ & $8.01 \pm 1.2$ & $8.04 \pm 1.5$ & $7.87 \pm 1.2$ & $7.24 \pm 1.6$ & $7.91 \pm 1.2$ & $7.78 \pm 1.3$ \\
\hline C18:3n-3 (ALA) & $2.10 \pm 0.5^{\mathrm{e}, \mathrm{f}}$ & $2.21 \pm 0.5^{\mathrm{e}, \mathrm{f}}$ & $1.98 \pm 0.4^{\mathrm{e}}$ & $1.94 \pm 0.5^{\mathrm{e}}$ & $0.52 \pm 0.2^{\mathrm{a}, \mathrm{b}, \mathrm{c}, \mathrm{d}, \mathrm{f}, \mathrm{g}, \mathrm{h}}$ & $1.22 \pm 0.3^{\mathrm{a}, \mathrm{b}, \mathrm{e}}$ & $1.79 \pm 0.2^{\mathrm{e}}$ & $1.42 \pm 0.3^{\mathrm{e}}$ \\
\hline C20:4n-6 (AA) & $7.41 \pm 0.5^{\mathrm{b}, \mathrm{d}, \mathrm{e}, \mathrm{f}}$ & $6.04 \pm 0.3^{\mathrm{a}, \mathrm{c}, \mathrm{e}, \mathrm{f}}$ & $7.84 \pm 0.5^{\mathrm{b}, \mathrm{d}, \mathrm{e}, \mathrm{f}}$ & $6.14 \pm 0.3^{\mathrm{a}, \mathrm{c}, \mathrm{e}, \mathrm{f}}$ & $4.05 \pm 0.2^{\mathrm{a}, \mathrm{b}, \mathrm{c}, \mathrm{d}, \mathrm{f}, \mathrm{g}, \mathrm{h}}$ & $5.76 \pm 0.4^{\mathrm{a}, \mathrm{c}, \mathrm{e}, \mathrm{g}, \mathrm{h}}$ & $6.98 \pm 0.4^{\mathrm{b}, \mathrm{d}, \mathrm{e}, \mathrm{f}}$ & $7.01 \pm 0.5 \mathrm{~b}, \mathrm{~d}, \mathrm{e}, \mathrm{f}$ \\
\hline C20:5n-3 (EPA) & $1.10 \pm 0.2^{b, d, e, g}$ & $3.15 \pm 0.5^{\mathrm{a}, \mathrm{c}, \mathrm{e}, \mathrm{f}, \mathrm{g}, \mathrm{h}}$ & $1.31 \pm 0.3^{\mathrm{b}, \mathrm{d}, \mathrm{e}, \mathrm{g}}$ & $3.32 \pm 0.4^{\mathrm{a}, \mathrm{c}, \mathrm{e}, \mathrm{f}, \mathrm{f}, \mathrm{g}, \mathrm{h}}$ & $0.21 \pm 0.01 \mathrm{a}, \mathrm{b}, \mathrm{c}, \mathrm{d}, \mathrm{f}, \mathrm{g}, \mathrm{h}$ & $0.94 \pm 0.2 \mathrm{~b}, \mathrm{~d}, \mathrm{e}, \mathrm{g}, \mathrm{h}$ & $0.58 \pm 0.1 \mathrm{a}, \mathrm{b}, \mathrm{c}, \mathrm{d}, \mathrm{d}, \mathrm{e}, \mathrm{f}, \mathrm{h}$ & $1.50 \pm 0.3^{b, d, e, f, g}$ \\
\hline C22:6n-3 (DHA) & $2.58 \pm 0.3^{b, d, e, g}$ & $3.76 \pm 0.5^{\mathrm{a}, \mathrm{c}, \mathrm{e}, \mathrm{f}, \mathrm{g}, \mathrm{h}}$ & $2.81 \pm 0.4^{\mathrm{b}, \mathrm{d}, \mathrm{e}, \mathrm{g}}$ & $3.88 \pm 0.4 \mathrm{a}, \mathrm{c}, \mathrm{e}, \mathrm{f}, \mathrm{g}, \mathrm{h}$ & 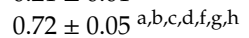 & $2.14 \pm 0.2^{b, d, e}$ & $1.95 \pm 0.2^{\mathrm{a}, \mathrm{b}, \mathrm{c}, \mathrm{d}, \mathrm{e}, \mathrm{h}}$ & $2.64 \pm 0.3^{b, d, e, g}$ \\
\hline Total SFAs & $47.0 \pm 4.3^{\mathrm{e}}$ & $43.7 \pm 4.6^{\mathrm{e}}$ & $45.1 \pm 4.9^{\mathrm{e}}$ & $44.9 \pm 4.7 \mathrm{e}$ & $58.9 \pm 5.8^{\mathrm{a}, \mathrm{b}, \mathrm{c}, \mathrm{d}}$ & $51.8 \pm 5.4$ & $52.9 \pm 5.0$ & $50.1 \pm 4.4 \mathrm{e}^{\mathrm{e}}$ \\
\hline Total MUFAs & $29.3 \pm 2.9$ & $30.4 \pm 3.1$ & $31.4 \pm 3.2$ & $30.8 \pm 2.7$ & $27.1 \pm 3.1$ & $29.3 \pm 2.5$ & $24.7 \pm 3.1$ & $27.6 \pm 3.3$ \\
\hline Total PUFAs & $23.7 \pm 2.5^{\mathrm{e}, \mathrm{f}}$ & $25.9 \pm 2.7^{\mathrm{e}, \mathrm{f}}$ & $23.5 \pm 2.4 \mathrm{e}, \mathrm{f}$ & $24.3 \pm 2.1^{\mathrm{e}, \mathrm{f}}$ & $14.0 \pm 1.4^{\mathrm{a}, \mathrm{b}, \mathrm{c}, \mathrm{d}, \mathrm{f}, \mathrm{g}, \mathrm{h}}$ & $18.9 \pm 1.8^{\mathrm{a}, \mathrm{b}, \mathrm{c}, \mathrm{d}, \mathrm{e}}$ & $22.4 \pm 2.4^{\mathrm{e}}$ & $22.3 \pm 2.2^{\mathrm{e}}$ \\
\hline Total LCPUFAs & $12.9 \pm 0.4$ e,f,g,h & $13.7 \pm 0.5^{\mathrm{e}, \mathrm{f}, \mathrm{g}, \mathrm{h}}$ & $13.1 \pm 0.3^{\mathrm{e}, \mathrm{f}, \mathrm{g}, \mathrm{h}}$ & $14.0 \pm 0.4 \mathrm{e}, \mathrm{f}, \mathrm{g}, \mathrm{h}$ & 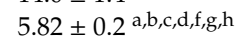 & $9.76 \pm 0.4^{\mathrm{a}, \mathrm{b}, \mathrm{c}, \mathrm{d}, \mathrm{e}, \mathrm{h}}$ & $10.4 \pm 0.44^{\mathrm{a}, \mathrm{b}, \mathrm{c}, \mathrm{d}, \mathrm{e}, \mathrm{h}}$ & $11.7 \pm 0.3^{\mathrm{a}, \mathrm{b}, \mathrm{c}, \mathrm{d}, \mathrm{e}, \mathrm{f}, \mathrm{g}}$ \\
\hline Total n-6 LCPUFAs & $8.78 \pm 0.3^{b, d, e, f, g, h}$ & $6.14 \pm 0.2^{\mathrm{a}, \mathrm{c}, \mathrm{e}, \mathrm{g}, \mathrm{h}}$ & $9.04 \pm 0.4^{\mathrm{b}, \mathrm{d}, \mathrm{e}, \mathrm{f}, \mathrm{g}, \mathrm{h}}$ & $6.46 \pm 0.2^{\mathrm{a}, \mathrm{a}, \mathrm{e}, \mathrm{g}, \mathrm{h}}$ & $4.42 \pm 0.2^{a}, \mathrm{~b}, \mathrm{~b}, \mathrm{~d}, \mathrm{f}, \mathrm{g}, \mathrm{h}$ & $6.27 \pm 0.4 \mathrm{a}, \mathrm{c}, \mathrm{e}$ & $7.72 \pm 0.5^{\mathrm{a}, \mathrm{c}, \mathrm{e}}$ & $7.22 \pm 0.5 \mathrm{a}, \mathrm{c}, \mathrm{e}$ \\
\hline Total n-3 LCPUFAs & $4.12 \pm 0.2^{\mathrm{b}, \mathrm{d}, \mathrm{e}, \mathrm{f}, \mathrm{g}}$ & $7.56 \pm 0.3 \mathrm{a}, \mathrm{c}, \mathrm{e}, \mathrm{f}, \mathrm{g}, \mathrm{h}$ & $4.06 \pm 0.2^{b, d, e, f, g}$ & $7.54 \pm 0.44^{\mathrm{a}, \mathrm{c}, \mathrm{e}, \mathrm{f}, \mathrm{g}, \mathrm{h}}$ & $1.40 \pm 0.04 \mathrm{a}, \mathrm{b}, \mathrm{c}, \mathrm{d}, \mathrm{f}, \mathrm{g}, \mathrm{h}$ & $3.49 \pm 0.2^{a, b, c, d, e, g, h}$ & $2.68 \pm 0.1 \mathrm{a}, \mathrm{b}, \mathrm{c}, \mathrm{d}, \mathrm{d}, \mathrm{e}, \mathrm{f}, \mathrm{h}$ & $4.48 \pm 0.04$ b,d,e,f,g \\
\hline n-6/n-3 LCPUFAs ratio & $2.13 \pm 0.1^{b, d, e, f, g, h}$ & $0.81 \pm 0.0$ a,c,e, $, \mathrm{f}, \mathrm{g}, \mathrm{h}$ & $2.22 \pm 0.1^{b, d, e, f, g, h}$ & $0.86 \pm 0.03^{\mathrm{a}, \mathrm{c}, \mathrm{e}, \mathrm{f}, \mathrm{g}, \mathrm{h}}$ & $3.15 \pm 0.2^{\mathrm{a}, \mathrm{b}, \mathrm{b}, \mathrm{d}, \mathrm{d}, \mathrm{f}, \mathrm{g}, \mathrm{h}}$ & $1.80 \pm 0.03 \mathrm{a}, \mathrm{b}, \mathrm{c}, \mathrm{d}, \mathrm{e}, \mathrm{g}$ & $2.88 \pm 0.2^{a, b, c, d, e, f, h}$ & $1.61 \pm 0.1^{\mathrm{a}, \mathrm{b}, \mathrm{c}, \mathrm{d}, \mathrm{e}, \mathrm{g}}$ \\
\hline
\end{tabular}

Values are expressed as mean \pm standard deviation (SD). Eight to ten mice were included in each experimental group. Significant differences between the groups are indicated by the letter identifying each group, namely, (a), CD + saline; (b) CD + EPA; (c) CD + HT; (d) CD + EPA + HT; (e), HFD + saline; (f) HFD + EPA, (g) HFD + HT; and (h), HFD + EPA + HT, $p<$ 0.05 , two-way analysis of variance (ANOVA) followed by Tukey's post-test. Fatty acid methyl esters (FAME). Saturated fatty acids (SFAs) correspond to C12:0, C14:0, C16:0 and C18:0. Monounsaturated fatty acids (MUFAs) correspond to C14:1n-7, C16:1n-7 and C18:1n-9. Polyunsaturated fatty acids (PUFAs) correspond to C18:2n-6, C18:3n-3, C20:4n-6, C20:5n-3, C22:5n-3 and C22:6n-3, n-6 LCPUFAs are C20:4n-6 and C22:5n-3, n-3 LCPUFAs are C20:5n-3, C22:5n-3 and C22:6n-3, n-6/n-3 LCPUFAs ratio: C20:4n-6/(C20:5n-3 + C22:5n-3 + C22:6n-3). 


\subsection{HFD-Induced EWAT Oxidative Stress Enhancement is Normalized by EPA and HT Co-Administration}

The treatments showed no effect on any of the parameters associated with oxidative stress evaluated in EWAT of CD-fed mice (Figure 1). HFD feeding induced oxidative stress in EWAT, as evidenced by the increase in markers of oxidative damage TBARS (Figure 1A), F2-isoprostanes (Figure 1B) and protein carbonyls (Figure 1C) accompanied by alteration of the glutathione status (Figure 1D-G). Supplementation with EPA alone only reduced levels of protein carbonyls (Figure 1C), without improving the rest of the parameters evaluated in EWAT of HFD animals (Figure 1A,B,D-G). When HT was given alone, TBARS and F2-isoprostanes levels were reduced (Figure 1A,B), and the content of protein carbonyls (Figure 1C) and glutathione levels (Figure 1D-G) were normalized, reaching control values. Notably, the administration of both supplements, EPA + HT, prevented oxidative damage in all parameters evaluated and deterioration of glutathione status in EWAT of mice receiving the HFD (Figure 1A-D).

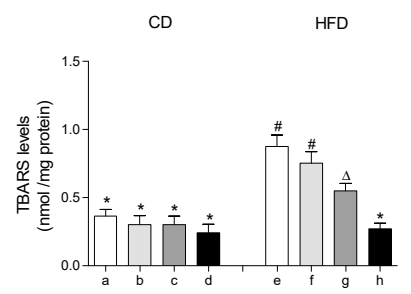

D

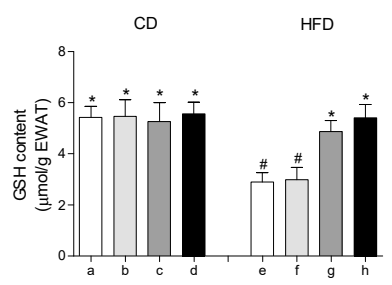

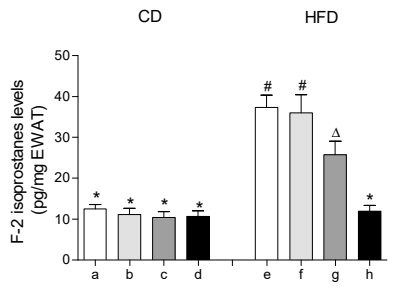

E

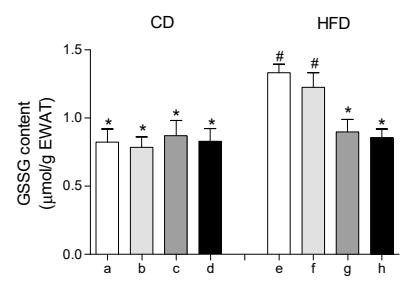

G

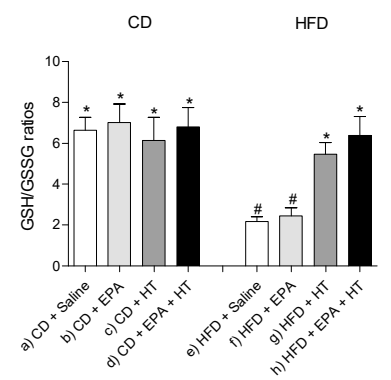

C

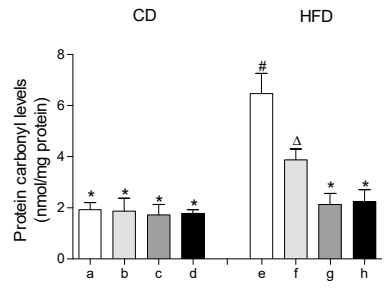

F

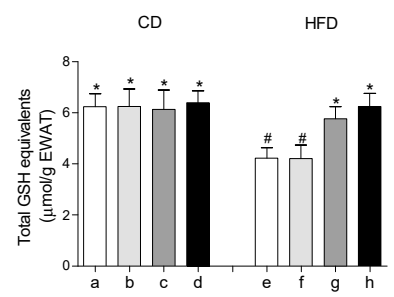

Figure 1. Thiobarbituric acid reactive substances (TBARS), (A) F-2 isoprostanes, (B) protein carbonyl groups, (C) reduced glutathione (GSH) content, (D) glutathione disulfide (GSSG) levels, (E) total GSH equivalents (GSH + 2GSSG) and (F) GSH/GSSG ratio (G) in epididymal white adipose tissue from mice subjected to control diet (CD) and high-fat diet (HFD) supplemented or not with eicosapentaenoic acid (EPA), hydroxytyrosol (HT) or EPA + HT. Values are expressed as mean $\pm \mathrm{SD}$. Eight to ten mice were included in each experimental group. Groups that do not share the same superscript symbol were significantly different among them, namely, (a), CD + saline; (b) CD + EPA; (c) CD + HT; (d) CD + EPA + HT; (e), HFD + saline; (f) HFD + EPA, (g) HFD + HT; and (h), HFD + EPA + HT, according to two-way analysis of variance (ANOVA) followed by the Tukey's post-test, $p<0.05$. $\left({ }^{*}\right)$, does not differ from (a) group; (\#), differ from (a) group; $(\Delta)$, differ from (a) and (b) groups.

The fall in the mRNA levels and the DNA binding capacity impaired of the cytoprotective transcription factor Nrf2 in EWAT of HFD-fed mice were partially recovered when animals received EPA or HT supplementation (Figure 2A,B). Likewise, the HFD-induced diminution in the expression levels 
of its downstream genes glutamate-cysteine ligase (GCL) (Figure 2C) and glutathione-S-transferase (GST) (Figure 2D) involved in glutathione metabolism were equally improved by EPA or HT. The supplementation with EPA plus HT in HFD mice showed an even greater effect, restoring the expression levels of Nrf2, GCL and GST to control values, and normalizing the DNA binding activity of Nrf2 (Figure 2A-D). A similar behavior was observed in the activity of antioxidant enzymes CAT (Figure 2E), SOD (Figure 2F), GR (Figure 2G) and GPX (Figure 2H) in EWAT of HFD-mice that received EPA, HT or both supplements, which were partially enhanced by EPA or HT after reduction by HFD and totally recovered by combined EPA and HT (Figure 2D-H).

A

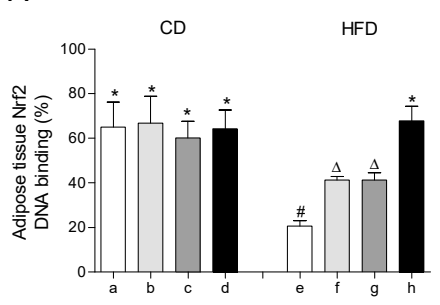

D

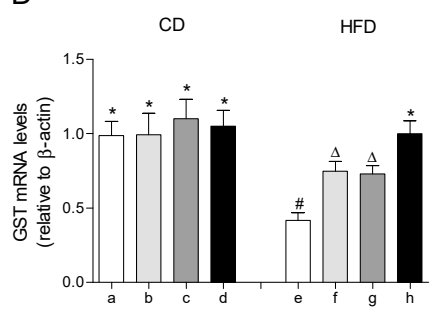

B

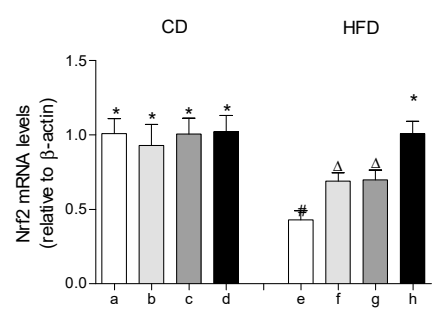

E

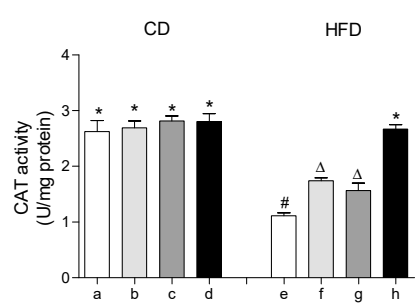

C

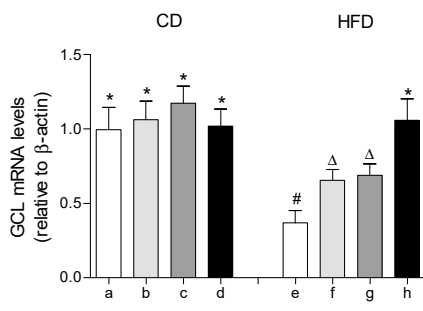

F CD $\quad H F D$

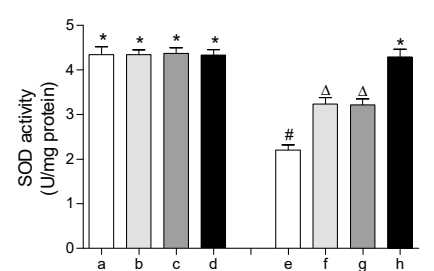

$C D$

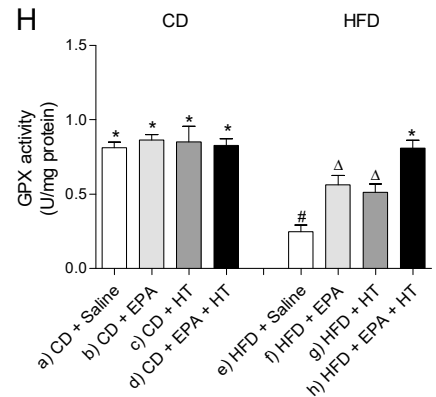

Figure 2. Nuclear factor E2-related factor 2 (Nrf2) DNA binding, (A) Nrf2 mRNA levels, (B) mRNA levels of glutamate-cysteine ligase (GCL) (C) and glutathione-S-transferase (GST), (D) and activity of catalase (CAT), (E) superoxide dismutase (SOD), (F) glutathione peroxidase (GPX), (G) and glutathione reductase (GR) (H) in epididymal white adipose tissue from mice subjected to control diet (CD) and high-fat diet (HFD) supplemented or not with eicosapentaenoic acid (EPA), hydroxytyrosol (HT) or $\mathrm{EPA}+\mathrm{HT}$. Values are expressed as mean $\pm \mathrm{SD}$. Eight to ten mice were included in each experimental group. Groups that do not share the same superscript symbol were significantly different among them, namely, (a), CD + saline; (b) CD + EPA; (c) CD + HT; (d) CD + EPA + HT; (e), HFD + saline; (f) HFD + $\mathrm{EPA},(\mathrm{g}) \mathrm{HFD}+\mathrm{HT}$; and (h), HFD + EPA + HT, according to two-way analysis of variance (ANOVA) followed by the Tukey's post-test, $p<0.05$. $\left({ }^{*}\right)$, does not differ from (a) group; (\#), differ from (a) group and does not differ from (e) group; $(\Delta)$, differ from (a) and (b) groups.

3.4. HFD-Induced Upregulation of SREBP-1c System and Downregulation of PPAR- $\gamma$ Pathway in EWAT are Partially Recovered by the Combined EPA and HT Protocol

The EWAT of mice subjected to HFD exhibit a significant increase in DNA-binding activity (Figure 3A) and mRNA levels of SREBP-1c (Figure 3B). The activation of SREBP-1c was accompanied 
by higher gene expression and activities of lipogenic enzymes ACC (Figure 3E,F) and FAS (Figure 3C,D) and higher activities of G6PD (Figure 3G) and ME (Figure 3H) compared to CD values. In addition, mRNA levels of enzyme LPL were decreased, whereas their activity increased in EWAT of HFD-fed mice (Figure 4D,E). The administration of EPA or HT supplied alone to HFD mice led to a partial recovery of all the lipogenic parameters evaluated in EWAT (Figure 3) including the LPL enzyme (Figure 4D,E), showing comparable results with both supplements, except for the FAS activity (Figure 3G). The effect of EPA and HT given together led to a significantly greater recovery of SREBP-1c (Figure 3A,B) and its target genes FAS, ACC, G6PD, ME (Figure 3C-H) and LPL (Figure 4D,E), although without achieving values observed in EWAT of $C D$ mice.

A

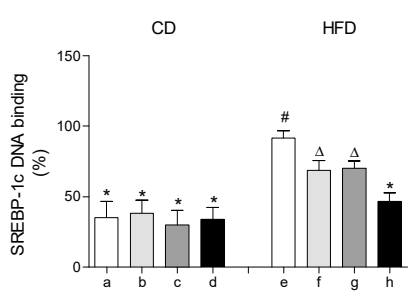

D

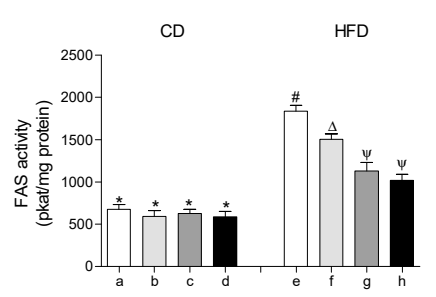

B

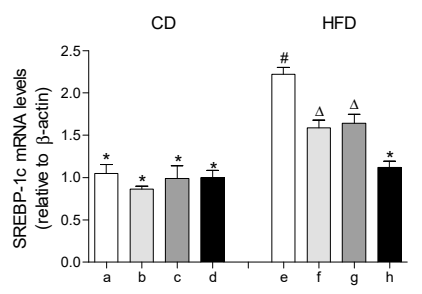

E

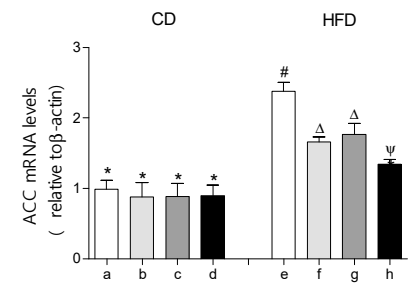

C

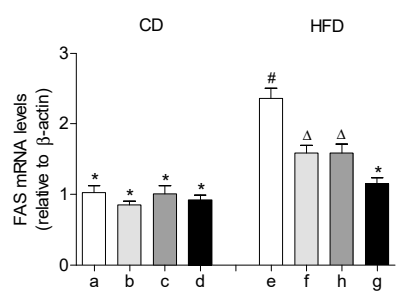

$\mathrm{F}$

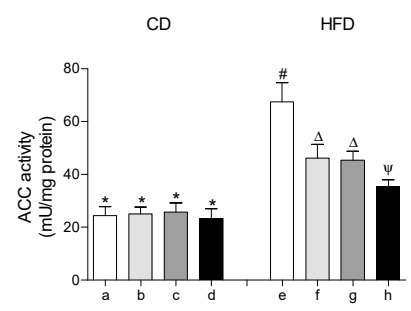

G

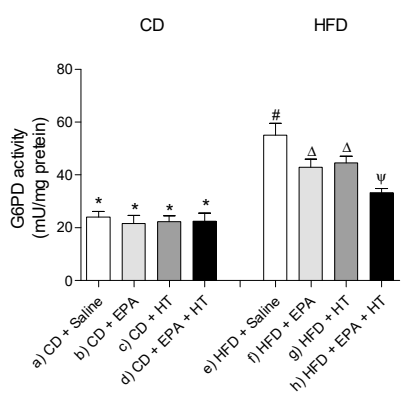

$\mathrm{H}$

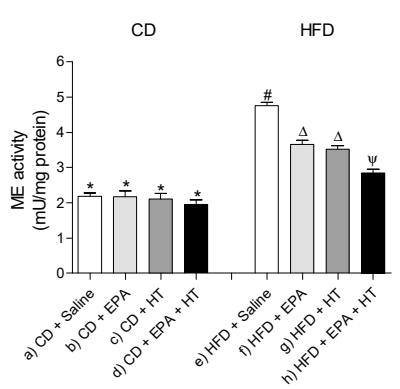

Figure 3. Sterol regulatory element-binding protein-1c (SREBP-1c) DNA-binding, (A) SREBP-1 mRNA levels, (B) fatty acid synthase (FAS) mRNA content, (C) FAS activity, (D) acetyl-CoA carboxylase (ACC) mRNA levels, (E) ACC activity, (F) glucose-6-phosphate dehydrogenase (G6PD) activity, (G) and malic enzyme (ME) activity $(\mathbf{H})$ in epididymal white adipose tissue from mice subjected to control diet (CD) and high-fat diet (HFD) supplemented or not with eicosapentaenoic acid (EPA), hydroxytyrosol (HT) or EPA + HT. Values are expressed as mean \pm SD. Eight to ten mice were included in each experimental group. Groups that do not share the same superscript symbol were significantly different among them, namely, (a), CD + saline; (b) CD + EPA; (c) CD + HT; (d) CD + EPA + HT; (e), HFD + saline; (f) HFD + EPA, (g) HFD + HT; and (h), HFD + EPA + HT, according to two-way analysis of variance (ANOVA) followed by the Tukey's post-test, $p<0.05 .\left({ }^{*}\right)$, does not differ from (a) group; (\#), differ from (a) group; $(\Delta)$, differ from (a) and (e) groups; ( $\Psi$ ), differs from groups (a), (e) and those marked with the symbol $\Delta$. 


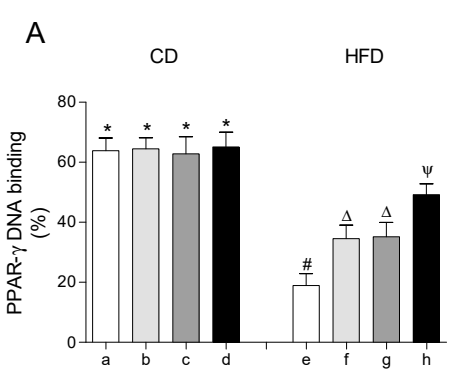

B

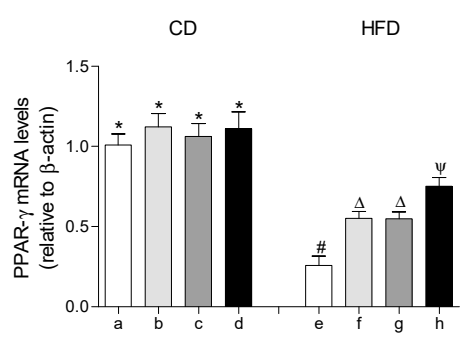

C

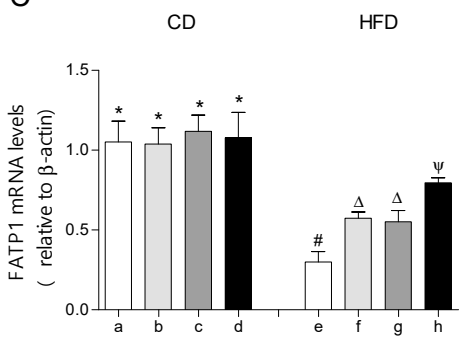

D $\quad$ CD

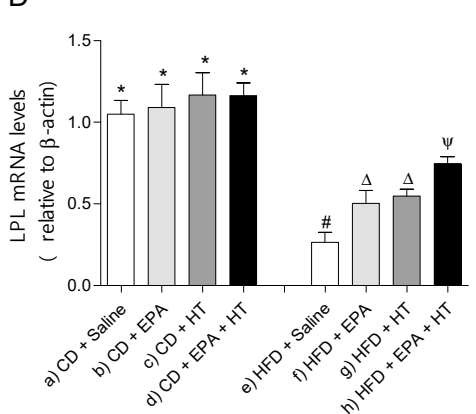

E

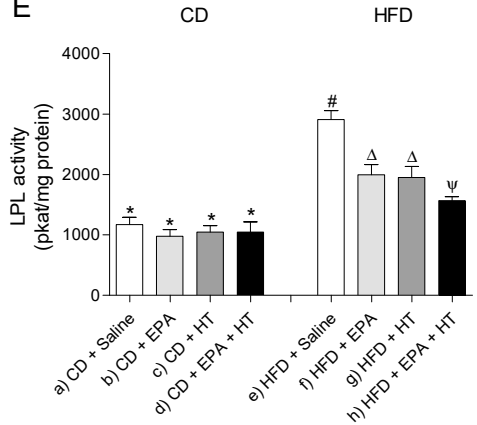

Figure 4. Peroxisome proliferator-activated receptor-gamma (PPAR- $\gamma$ ) DNA-binding, (A) mRNA levels of PPAR- $\gamma,($ B) fatty acid transport protein 1 (FATP1), (C) lipoprotein lipase (LPL) (D) and LPL activity (E) in epididymal white adipose tissue from mice subjected to control diet (CD) and high-fat diet (HFD) supplemented or not with eicosapentaenoic acid (EPA), hydroxytyrosol (HT) or EPA + HT. Values are expressed as mean \pm SD. Eight to ten mice were included in each experimental group. Groups that do not share the same superscript symbol were significantly different among them, namely, (a), CD + saline; (b) CD + EPA; (c) CD + HT; (d) CD + EPA + HT; (e), HFD + saline; (f) HFD + EPA, (g) HFD + HT; and (h), HFD + EPA + HT, according to two-way analysis of variance (ANOVA) followed by the Tukey's post-test, $p<0.05$. $\left({ }^{*}\right)$, does not differ from (a) group; (\#), differ from (a) group; $(\Delta)$, differ from (a) and (e) groups; ( $\Psi)$, differs from groups (a), (e) and those marked with the symbol $\Delta$.

The DNA-binding activity (Figure 4A) and mRNA levels of PPAR- $\gamma$ (Figure 4B) and fatty acid transport protein 1 (FATP1) (Figure 4C) were decreased in EWAT of HFD-fed mice compared to the control group. Supplementation whit EPA or HT alone similarly improved the activity and mRNA levels of PPAR- $\gamma$ and gene expression of FATP1. Moreover, EPA plus HT supplementation led to a significant increase in the binding activity and gene expression of PPAR- $\gamma$ and in the gene expression of the FATP1 transporter compared to groups that received EPA or HT, without reaching CD values (Figure $4 \mathrm{~A}-\mathrm{C}$ ). Mice subjected to $\mathrm{CD}$ with or without supplementation showed comparable results in all parameters evaluated in EWAT mentioned in Figures 3 and 4.

3.5. HFD-Induced Activation of Transcription Factor NF- $\kappa B$ and Expression of Target Genes Associated with Inflammation in EWAT are Diminished to Values over CD by the EPA Plus HT Protocol

The DNA-binding activity and the gene expression of the transcription factor NF- $\mathrm{KB}$ were significantly increased in EWAT of mice fed with the HFD compared to those that received the CD (Figure 5A,B). In parallel, the mRNA levels of the pro-inflammatory cytokine tumor necrosis factor alpha (TNF- $\alpha$ ) and interleukin 6 (IL-6) were increased in EWAT of HFD-fed mice (Figure 5C,D). In the HFD-fed mice that received EPA or HT, the DNA-binding activity of NF- $\mathrm{kB}$ and the mRNA levels of NF- $\mathrm{KB}$, TNF- $\alpha$ and IL-6 were moderately improved, with comparable results between both groups, respectively. The group that received EPA plus HT showed a significantly greater decrease in these values in comparison to the groups supplemented with EPA or HT alone, without reaching those of the control group (Figure 5). 
A

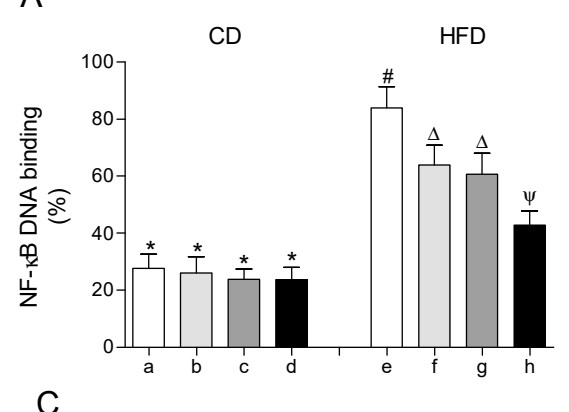

C

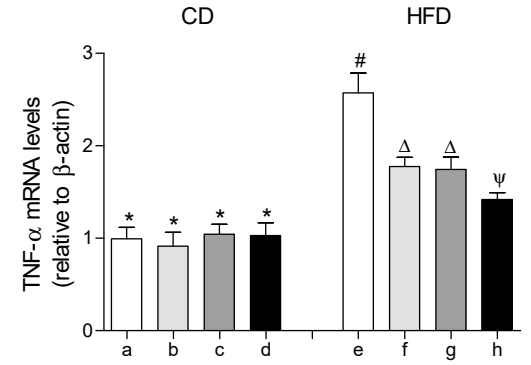

B

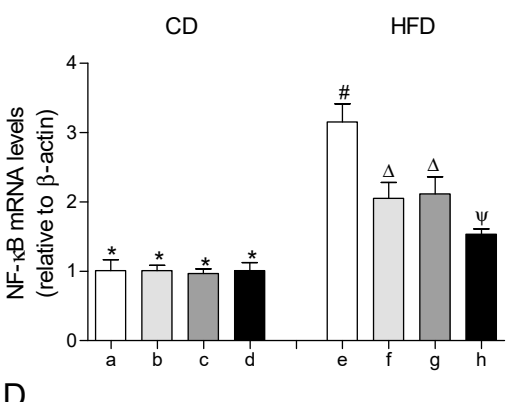

D

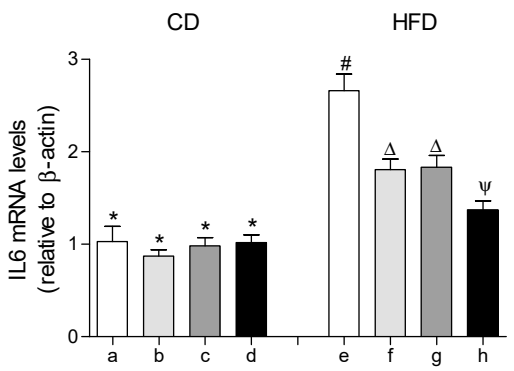

Figure 5. Nuclear factor erythroid 2-related factor 2 (NF-kB) DNA-binding, (A) mRNA levels of NF- $\mathrm{kB},(\mathbf{B})$ mRNA levels of tumor necrosis factor (TNF- $\alpha$ ), (C) and mRNA levels of interleukin 6 (IL-6) (D) in epididymal white adipose tissue from mice subjected to control diet (CD) and high-fat diet (HFD) supplemented or not with eicosapentaenoic acid (EPA), hydroxytyrosol (HT) or EPA + HT. Groups that do not share the same superscript symbol were significantly different among them, namely, (a), CD + saline; (b) CD + EPA; (c) CD + HT; (d) CD + EPA + HT; (e), HFD + saline; (f) HFD + EPA, (g) HFD + HT; and (h), HFD + EPA + HT, according to two-way analysis of variance (ANOVA) followed by the Tukey's post-test, $p<0.05$. $\left({ }^{*}\right)$, does not differ from (a) group; (\#), differ from (a) group; $(\Delta)$, differ from (a) and (e) groups; ( $\Psi)$, differs from groups (a), (e) and those marked with the symbol $\Delta$.

\section{Discussion}

Diet-induced obesity (DIO) in mice represents a model of choice for preclinical nutritional studies [33-35]. HFD feeding comprising 60\% of the total calories as fat for 12 weeks achieved significant BW gain with concomitant liver and WAT dysfunction. In the case of the liver, HFD induced steatosis in association with n-3 LCPUFAs depletion, oxidative stress with loss of antioxidant defenses [20], a pro-inflammatory state without histological inflammation [17] and a decline in mitochondrial energy metabolism [18]. The drastic metabolic alterations induced by HFD are evidenced by the deranged levels of (i) serum-free fatty acids, the adipokines adiponectin and leptin, and the cytokines TNF- $\alpha$ and IL-6, which are associated with IR development [13], (ii) the development of adipocyte hypertrophy and enhancement in total tissue mass, with higher cell size and TAG content and lower cell number per $g$ of tissue over control values and (iii) the derangement of metabolic parameters in WAT favoring oxidative stress, lipogenesis, mitochondrial dysfunction and inflammation, features that agree with a previous report [13] and resemble those elicited in the liver $[17,18,20]$.

Metabolic alterations can be prevented or attenuated by the combination of active substances, including n-3 LCPUFAs, antioxidants and insulin sensitizers, as reported for DHA, EPA, HT, rosiglitazone and astaxantin $[17,18,36,37]$. In the present study, EPA and HT co-administration diminished BW gain in HFD-fed mice over CD animals, an effect that was accompanied by a partial WAT reduction and EWAT cellularity preservation similar to those observed in the separate supplementations. In agreement with these findings, replacement of dietary lipids in a HFD by $1 \%$ to $12 \%(w t / w t)$ of EPA plus DHA (DHA > EPA) limited the BW gain and EWAT accumulation in C57BL/6J HFD-fed mice, with similar results being found by replacing a HFD rich in ALA or linoleic acid (C18:2n-6, LA) by mixtures of EPA and DHA at different ratios [38]. The effect of EPA on obesity may be partly mediated by binding to $G$ protein-coupled receptor 120 (GPR120) present in adipocytes and adipocyte tissue macrophages 
(ATMs) [39], thus regulating adipogenesis, glucose uptake, inflammation and insulin sensitivity [40]. EPA binding and activation of GPR120 promotes several effects, including (i) the interaction with PPAR- $\gamma$ that favors the differentiation of pre-adipocytes into white adipocytes [40], (ii) the binding to $\beta$-arrestin 2 and their interaction with transforming growth factor- $\beta$-activated kinase binding protein 1 (TAB1) that blocks NF- $\kappa B$ and $c$ Jun N-terminal kinase (JNK) functioning, with inhibition of the low-grade inflammation due to TNF- $\alpha$ and IL- 6 production by ATMs triggered by obesity [41] and (iii) the release of fibroblast growth factor 21 (FGF21), resulting in the promotion of brown adipose tissue (BAT) activity and WAT browning [42], with stimulation of mitochondrial energy metabolism depressed by HFD. It is important to note that EPA supplementation in HFD-fed mice upregulates the expression of PPAR- $\alpha$ and the micro-RNAs miR-455 and miR-129-5p in BAT [43]. These mediators enhance the expression of thermogenic markers that stimulate mitochondrial FAO and energy expenditure, pointing to BAT as a crucial anti-obesity target [43]. This contention is further supported by data showing that HT prevents visceral adipogenesis and decreased metabolic activity in BAT and WAT, induced by the exposure to fine particulate matter in mice [44]. The contribution of EPA to the induction of a healthy adipocyte phenotype may also involve its direct binding to PPAR- $\gamma$, whose expression and activity are diminished by HFD and partially rescued by EPA. Activation of PPAR- $\gamma$ induces adipogenesis as an important mechanism of WAT remodeling, which is accomplished through downregulation of SREBP-1c functioning $[9,39]$. The HT component of the combined protocol elicited diminution of WAT lipid accumulation by regulating genes related to lipogenesis and FAO, but mainly due to the improvement of the cellular antioxidant status that preserves n-3 LCPUFAs loss by HFD $[13,45]$. The latter mechanism of HT action is exerted directly as a free-radical scavenger or by recovering the Nrf2 system that induces antioxidant enzymes (SOD, CAT, GPX) and those associated with glutathione repletion (GCL, GR) or utilization (GST). At the doses used in our study, the combined EPA and HT administration did not exhibit more potentiated effects on WAT morphology parameters and lipid content than those of the individual compounds. However, the parameters related to WAT metabolic dysfunction are diminished in an additive manner, considering that the specific effects exerted by EPA or HT exhibit convergence in the combined protocol.

In agreement with previous data, WAT expansion in HFD-fed mice led to a significant decrease of serum levels of adiponectin and an increase in those of leptin [13], which are correlated with IR [46] and are partially restored by EPA and HT single or combined supplementations. In this context, the administration of EPA is known to enhance the gene expression and release of adiponectin from adipocytes, either in EWAT from HFD-fed mice [47] or in lean and overweight rats [48], thus evidencing the insulin-sensitizing properties of EPA $[17,47,48]$. Interestingly, the administration of EPA to obese subjects elevated adiponectin levels without altering BW, suggesting direct involvement of EPA on adiponectin secretion [49]. This conclusion is also supported by EPA supplementation to HFD-fed mice showing maintenance of normal adiponectin levels without diminishing adiposity, pointing to adiponectin as a major factor in EPA-mediated prevention and reversal of IR [50]. Regarding the molecular mechanisms underlying these effects, EPA increase the expression of adiponectin through binding to PPAR- $\gamma$, promoting the expression of FGF21 and the activation of AMP-activated protein kinase (AMPK), the energy sensor that favors FAO over lipogenesis $[15,42,47,51]$. The EPA-induced AMPK activation by phosphorylation in 3T3-L1 adipocytes [51] is in agreement with in vivo studies showing that $n-3$ LCPUFAs increase the content of WAT AMPK $\alpha 1$ subunit and its phosphorylation status, in association with an enhancement in adiponectin levels [52,53]. In the case of leptin, the enhancement in its serum levels by HFD feeding in mice and their diminution by EPA (Table 2D) agree with studies in humans and rodents [54], suggesting that improved leptin sensitivity concomitantly with decreased production would be secondary to the anti-inflammatory effect of n-3 LCPUFAs [55]. Regarding HT, several preclinical studies reported higher circulating levels of adiponectin with lower leptin values than controls achieved by this polyphenol $[13,24,45,56]$.

Improved expression and activity of the PPAR- $\gamma$ gene by co-administration of EPA and HT also leads to improved expression of the target genes FATP1 and LPL, an effect that is additive 
over the individual supplementations after HFD feeding. In particular, upregulation of WAT FATP1 may contribute to the smaller healthy phenotype of adipocytes due to its FA transport function across cell membranes and its acyl-CoA synthase activity to activate FAs for further utilization [57]. LPL, however, exhibited opposite changes regarding its mRNA expression and activity, which may be related to different mechanisms regulating LPL at transcriptional and post-translational levels [13]. The small healthy adipocyte phenotype is also associated with downregulation of key lipogenic genes in EPA-HT-HFD-treated mice. These include genes coding for transcription factor SREBP-1c that controls the expression of the lipogenic enzymes ACC and FAS and the reduced nicotinamide adenine dinucleotide phosphate (NADPH) generating systems G6PD and ME that are required for FA synthesis. The anti-lipogenic effect of the EPA plus HT protocol is related to the repletion of $n-3$ LCPUFAs due to lower oxidative deterioration by the normoxic state achieved, which downregulates lipogenic SREBP-1c functioning [18]. This contention is supported by previous studies showing (i) diminution in the lipogenic enzyme activities in WAT of sucrose-rich diet-fed rats subjected to fish oil administration [58], (ii) the decrease in the expression of the lipogenic enzyme glycerol-3-phosphate dehydrogenase in EPA-treated 3T3-L1 adipocytes [50] and (iii) the diminished SREBP-1c functioning with prevention of DIO in adipocytes from HFD-fed mice supplemented with EPA [59]. Additionally, HFD-induced prolipogenic state in EWAT with n-3 LCPUFAs depletion are attenuated by HT supplementation, which may preserve the activity of delta-5 and delta- 6 desaturases that contribute to n-3 LCPUFAs synthesis, as previously reported in the liver [24], downregulate SREBP-1c or reduce oxidative stress [13]. A major hallmark in the expansion of WAT in obesity is the enhancement in the adipocyte oxidative stress status, which underlies significant mitochondrial dysfunction. This is evidenced by the decreases in the activities of the carboxylic acid cycle regulatory enzyme citrate synthase and the respiratory chain complexes I and II [60], a condition that favors energy deprivation and mitochondrial reactive oxygen species (ROS) production, thus contributing to the attainment of a hypertrophic adipocyte phenotype [61,62]. Data presented indicate that HFD coupled to EPA plus HT supplementation totally avoided oxidative damage to biomolecules and the depletion of antioxidant systems in WAT. This finding mainly relies on the HT component of the combined protocol and may effectively contribute to the hypertrophic to the small healthy adipocyte phenotype transition observed. The reduction of the enlarged WAT in obesity achieved by EPA plus HT is in agreement with studies on the antioxidant behavior exerted by EPA, DHA and the carotenoid astaxantin, alone or in combinations in HepG2-C8 cells [37], EPA and DHA in 3T3-L1 adipocytes and Schwann cells [63,64] or in human macrophages [65], with responses exhibiting synergism. The EPA plus HT protocol also improved the pro-inflammatory status of WAT in HFD-fed mice in an additive manner. Possible mechanisms involved include: (i) disruption of the SFA inflammatory signal in Toll-like receptor 4 that activate NF- $\kappa B$, (ii) PPAR- $\gamma$ activation that interferes with the translocation of NF- $\mathrm{BB}$ to the nucleus, (iii) binding to GPR120 and prevention of NF-KB activation, (iv) decreased pro-inflammatory adipokine expression and macrophage accumulation, promoting repolarization towards the $\mathrm{M} 2$ anti-inflammatory phenotype by SPMs $[9,66,67]$ and (v) preservation of WAT n-3 LCPUFAs and downregulation of NF-кB by HT $[13,44]$.

\section{Conclusions}

HFD feeding in mice drastically impacts WAT functioning through the induction of hypertrophy and metabolic dysfunction. WAT hypertrophy involves higher cell size with TAG accumulation and lower cell number, which is related to EPA depletion that blunts PPAR- $\gamma$ operation. Metabolic dysfunction includes (i) upregulation of the lipogenic SREBP-1c system, (ii) loss of mitochondrial energy metabolism and (iii) downregulation of the antioxidant Nrf2 system, leading to oxidative stress and the secondary n-3 LCPUFAs depletion and activation of the redox-sensitive pro-inflammatory NF- $\mathrm{B}$ system. These effects of HFD were significantly diminished by the co-supplementation with EPA and HT additively, with resulting values being close or similar to those observed for the CD-fed animals. These observations strengthen the contention that EPA and HT co-administration is an important strategy to attenuate obesity-driven metabolic inflammation states. The data reported concerning PPAR- $\gamma$ upregulation by EPA and HT 
co-administration may also be of importance as a possible adjuvant therapy to diminish the severity of SARS-CoV-2 infection [68], considering the anti-inflammatory response involving PPAR- $\gamma$ binding to GPR120 and/or SPMs production from EPA and its product DHA, and the anti-fibrotic effect of PPAR- $\gamma$ [69].

Author Contributions: L.A.V. and R.V. designed the study. P.I., L.A.V. and R.V. analyzed and interpreted the data. A.E., F.E., S.S.-A., C.C., A.R., and R.V. performed the experiments. P.I., L.A.V., R.V. and T.M. structured and wrote the manuscript. All authors have read and agreed to the published version of the manuscript.

Funding: This research was funded by Initiation FONDECYT to Rodrigo Valenzuela grant number [11140174] and Regular FONDECYT to Alejandra Espinosa grant number [1181774].

Acknowledgments: The authors gratefully acknowledge Yolanda B. Lombardo, School of Biochemistry, University of Litoral, Santa Fe, Argentina, for the technical support received in her research group.

Conflicts of Interest: The authors declare no conflict to interest.

\section{References}

1. World Health Organization. Obesity and Overweight. Available online: https://www.who.int/news-room/factsheets/detail/obesity-and-overweight (accessed on 26 August 2020).

2. GBD 2015 Obesity Collaborators; Afshin, A.; Forouzanfar, M.H.; Reitsma, M.B.; Sur, P.; Estep, K.; Lee, A.; Marczak, L.; Mokdad, A.H.; Moradi-Lakeh, M.; et al. Health Effects of Overweight and Obesity in 195 Countries over 25 Years. N. Engl. J. Med. 2017, 377, 13-27. [CrossRef] [PubMed]

3. Chooi, Y.C.; Ding, C.; Magkos, F. The epidemiology of obesity. Metabolism 2019, 92, 6-10. [CrossRef] [PubMed]

4. Blüher, M. Obesity: Global epidemiology and pathogenesis. Nat. Rev. Endocrinol. 2019, 15, $288-298$. [CrossRef] [PubMed]

5. Fabbrini, E.; Sullivan, S.; Klein, S. Obesity and Nonalcoholic Fatty Liver Disease: Biochemical, Metabolic and Clinical Implications. Hepatology 2010, 51, 679-689. [CrossRef] [PubMed]

6. Rutkowski, J.M.; Stern, J.H.; Scherer, P.E. The cell biology of fat expansion. J. Cell Biol. 2015, 208, 501-512. [CrossRef] [PubMed]

7. Valenzuela, R.; Videla, L.A. The importance of the long-chain polyunsaturated fatty acid n-6/n-3 ratio in development of non-alcoholic fatty liver associated with obesity. Food Funct. 2011, 2, 644-648. [CrossRef] [PubMed]

8. Valenzuela, R.; Ortiz, M.; Hernandez-Rodas, M.C.; Videla, L.A. Targeting n-3 polyunsaturated fatty acids in non-alcoholic fatty liver disease. Curr. Med. Chem. 2020, 27, 5250-5272. [CrossRef]

9. Kuda, O.; Rossmeisl, M.; Kopecky, J. Omega-3 fatty acids and adipose tissue biology. Mol. Aspects Med. 2018, 64, 147-160. [CrossRef]

10. Magrone, T.; Spagnoletta, A.; Salvatore, R.; Magrone, M.; Dentamaro, F.; Russo, M.A.; Difonzo, G.; Summo, C.; Caponio, F.; Jirillo, E. Olive leaf extracts act as modulators of the human immune response. Endocr. Metab. Immune Disord. Drug Targets 2018, 18, 85-93. [CrossRef]

11. Echeverría, F.; Ortiz, M.; Valenzuela, R.; Videla, L.A. Hydroxytyrosol and cytoprotection: A projection for clinical interventions. Int. J. Mol. Sci. 2017, 18, 930. [CrossRef]

12. Valenzuela, R.; Illesca, P.; Echeverría, F.; Espinosa, A.; Rincón-Cervera, M.A.; Ortiz, M.; Hernández-Rodas, M.C.; Valenzuela, A.; Videla, L.A. Molecular adaptations underlying the beneficial effects of hydroxytyrosol in the pathogenic alterations induced by a high-fat diet in mouse liver: PPAR- $\alpha$ and Nrf2 activation, and NF-kB down-regulation. Food Funct. 2017, 8, 1526-1537. [CrossRef] [PubMed]

13. Illesca, P.; Valenzuela, R.; Espinosa, A.; Echeverría, F.; Soto-Alarcon, S.; Ortiz, M.; Videla, L.A. Hydroxytyrosol supplementation ameliorates the metabolic disturbances in white adipose tissue from mice fed a high-fat diet through recovery of transcription factors Nrf2, SREBP-1c, PPAR- $\gamma$ and NF- $k$ B. Biomed. Pharmacother. 2019, 109, 2472-2481. [CrossRef] [PubMed]

14. Valenzuela, R.; Videla, L.A. Crosstalk mechanisms in hepatoprotection: Thyroid hormone-docosahexaenoic acid (DHA) and DHA-extra virgin olive oil combined protocols. Pharmacol. Res. 2018, 132, 168-175. [CrossRef]

15. Valenzuela, R.; Videla, L.A. Impact of the co-administration of $n-3$ fatty acids and olive oil components in preclinical nonalcoholic fatty liver disease models: A mechanistic view. Nutrients 2020, 12, 499. [CrossRef] [PubMed] 
16. Talari, H.R.; Najafi, V.; Raygan, F.; Mirhosseini, N.; Ostadmohammadi, V.; Amirani, E.; Taghizadeh, M.; Hajijafari, M.; Shafabakhash, R.; Asemi, Z. Long-term Vitamin D and high-dose n-3 fatty acids' supplementation improve markers of cardiometabolic risk in type 2 diabetic patients with CHD. Br. J. Nutr. 2019, 122, 423-430. [CrossRef] [PubMed]

17. Echeverría, F.; Valenzuela, R.; Espinosa, A.; Bustamante, A.; Álvarez, D.; Gonzalez-Mañan, D.; Ortiz, M.; Soto-Alarcon, S.A.; Videla, L.A. Reduction of high-fat diet-induced liver proinflammatory state by eicosapentaenoic acid plus hydroxytyrosol supplementation: Involvement of resolvins RvE1/2 and RvD1/2. J. Nutr. Biochem. 2019, 63, 35-43. [CrossRef]

18. Echeverría, F.; Valenzuela, R.; Bustamante, A.; Álvarez, D.; Ortiz, M.; Espinosa, A.; Illesca, P.; Gonzalez-Mañan, D.; Videla, L.A. High-fat diet induces mouse liver steatosis with a concomitant decline in energy metabolism: Attenuation by eicosapentaenoic acid (EPA) or hydroxytyrosol (HT) supplementation and the additive effects upon EPA and HT co-administration. Food Funct. 2019, 10, 6170-6183. [CrossRef]

19. Valenzuela, R.; Barrera, C.; Espinosa, A.; Llanos, P.; Orellana, P.; Videla, L.A. Reduction in the desaturation capacity of the liver in mice subjected to high fat diet: Relation to LCPUFA depletion in liver and extrahepatic tissues. Prostaglandins Leukot. Essent. Fat. Acids 2015, 98, 7-14. [CrossRef]

20. Echeverría, F.; Valenzuela, R.; Bustamante, A.; Álvarez, D.; Ortiz, M.; Soto-Alarcon, S.A.; Muñoz, P.; Corbari, A.; Videla, L.A. Attenuation of high-fat diet-induced rat liver oxidative stress and steatosis by combined hydroxytyrosol-(HT-) eicosapentaenoic acid supplementation mainly relies on HT. Oxid. Med. Cell. Longev. 2018, 2018. [CrossRef]

21. Rodbell, M. Metabolism of Isolated Fat Cells:I. Effects on hormones on glucose metabolism and lipolysis. J. Biol. Chem. 1964, 239, 375-380. [PubMed]

22. Di Girolamo, M.; Mendlinger, S.; Fertig, J.W. A simple method to determine fat cell size and number in four mammalian species. Am. J. Physiol. 1971, 221, 850-858. [CrossRef] [PubMed]

23. Folch, J.; Lees, M.; Sloane Stanley, G.H. A simple method for the isolation and purification of total lipides from animal tissuers. J. Biol. Chem. 1957, 226, 497-509. [PubMed]

24. Valenzuela, R.; Echeverria, F.; Ortiz, M.; Rincón-Cervera, M.A.; Espinosa, A.; Hernández-Rodas, M.C.; Illesca, P.; Valenzuela, A.; Videla, L.A. Hydroxytyrosol prevents reduction in liver activity of $\Delta-5$ and $\Delta-6$ desaturases, oxidative stress, and depletion in long chain polyunsaturated fatty acid content in different tissues of high-fat diet fed mice. Lipids Health Dis. 2017, 16, 64. [CrossRef] [PubMed]

25. Bligh, E.G.; Dyer, W.J. A rapid method of total lipid extraction and purification. Can. J. Biochem. Physiol. 1959, 37, 911-917. [CrossRef]

26. Rahman, I.; Kode, A.; Biswas, S. Assay for quantitative determination of glutathione and glutathione disulfide levels using enzymatic recycling method. Nat. Protoc. 2006, 1, 3153-3165. [CrossRef]

27. Hernández-Rodas, M.C.; Valenzuela, R.; Echeverría, F.; Rincón-Cervera, M.A.; Espinosa, A.; Illesca, P.; Muñoz, P.; Corbari, A.; Romero, N.; Gonzalez-Mañan, D.; et al. Supplementation with Docosahexaenoic Acid and Extra Virgin Olive Oil Prevents Liver Steatosis Induced by a High-Fat Diet in Mice through PPAR- $\alpha$ and Nrf2 Upregulation with Concomitant SREBP-1c and NF-kB Downregulation. Mol. Nutr. Food Res. 2017, 61, 1700479. [CrossRef]

28. Wise, E.M.; Ball, E.G. Malic Enzyme and Lipogenesis. Proc. Natl. Acad. Sci. USA 1964, 52, 1255-1263. [CrossRef]

29. Llobera, M.; Montes, A.; Herrera, E. Lipoprotein lipase activity activity in liver of the rat fetus. Biochem. Biophys. Res. Commun. 1979, 91, 272-277. [CrossRef]

30. Lee, P.Y.; Costumbrado, J.; Hsu, C.Y.; Kim, Y.H. Agarose gel electrophoresis for the separation of DNA fragments. J. Vis. Exp. 2012, e3923. [CrossRef]

31. Pfaffl, M.W. A new mathematical model for relative quantification in real-time RT-PCR. Nucleic Acids Res. 2001, 29, e45. [CrossRef]

32. Livak, K.J.; Schmittgen, T.D. Analysis of relative gene expression data using real-time quantitative PCR and the 2- $\Delta \Delta \mathrm{CT}$ Method. Methods 2001, 25, 402-408. [CrossRef]

33. Nilsson, C.; Raun, K.; Yan, F.F.; Larsen, M.O.; Tang-Christensen, M. Laboratory animals as surrogate models of human obesity. Acta Pharmacol. Sin. 2012, 33, 173-181. [CrossRef]

34. Wong, S.K.; Chin, K.Y.; Suhaimi, F.H.; Fairus, A.; Ima-Nirwana, S. Animal models of metabolic syndrome: A review. Nutr. Metab. 2016, 13, 1-12. [CrossRef] [PubMed]

35. Chu, D.T.; Malinowska, E.; Jura, M.; Kozak, L.P. C57BL/6J mice as a polygenic developmental model of diet-induced obesity. Physiol. Rep. 2017, 5, 1-20. [CrossRef] [PubMed] 
36. Kuda, O.; Jelenik, T.; Jilkova, Z.; Flachs, P.; Rossmeisl, M.; Hensler, M.; Kazdova, L.; Ogston, N.; Baranowski, M.; Gorski, J.; et al. N-3 Fatty acids and rosiglitazone improve insulin sensitivity through additive stimulatory effects on muscle glycogen synthesis in mice fed a high-fat diet. Diabetologia 2009, 52, 941-951. [CrossRef] [PubMed]

37. Saw, C.L.L.; Yang, A.Y.; Guo, Y.; Kong, A.N.T. Astaxanthin and omega-3 fatty acids individually and in combination protect against oxidative stress via the Nrf2-ARE pathway. Food Chem. Toxicol. 2013, 62, 869-875. [CrossRef] [PubMed]

38. Ruzickova, J.; Rossmeisl, M.; Prazak, T.; Flachs, P.; Sponarova, J.; Vecka, M.; Tvrzicka, E.; Bryhn, M.; Kopecky, J. Omega-3 PUFA of marine origin limit diet-induced obesity in mice by reducing cellularity of adipose tissue. Lipids 2004, 39, 1177-1185. [CrossRef] [PubMed]

39. Im, D.S. FFA4 (GPR120) as a fatty acid sensor involved in appetite control, insulin sensitivity and inflammation regulation. Mol. Aspects Med. 2018, 64, 92-108. [CrossRef]

40. Song, T.; Yang, Y.; Zhou, Y.; Wei, H.; Pengs, J. GPR120: A critical role in adipogenesis, inflammation, and energy metabolism in adipose tissue. Cell. Mol. Life Sci. 2017, 74, 2723-2733. [CrossRef]

41. Oh, D.Y.; Talukdar, S.; Bae, E.J.; Imamura, T.; Morinaga, H.; Fan, W.; Li, P.; Lu, W.J.; Watkins, S.M.; Olefsky, J.M. GPR120 is an omega-3 fatty acid receptor mediating potent anti-inflammatory and insulin sensitizing effects. Cell 2010, 142, 687-698. [CrossRef]

42. Quesada-López, R.; Cereijo, J.V.; Turatsinze, A.; Planavila, M.; Cairó, A.; Gavaldà-Navarro, M.; Peyrou, R.; Moure, R.; Iglesias, M.; Giralt, M.; et al. The lipid sensor GPR120 promotes brown fat activation and FGF21 release from adipocytes. Nat. Comun. 2016, 7, 1-17. [CrossRef]

43. Pahlavani, M.; Wijayatunga, N.N.; Kalupahana, N.S.; Ramalingam, L.; Gunaratne, P.H.; Coarfa, C.; Rajapakshe, K.; Kottapalli, P.; Moustaid-Moussa, N. Transcriptic and microRNA analyses of gene meteorks regulated by eicosapentaenoic acid in brown adipose tissue of diet-induced obese mice. Biochim. Biophys. Acta Mol. Cell. Biol. Lipids. 2018, 1863, 1523-1531. [CrossRef] [PubMed]

44. Wang, N.; Ma, Y.; Liu, Z.; Liu, L.; Yang, K.; Wei, Y.; Liu, Y.; Chen, X.; Sun, X.; Wen, D. Hydroxytyrosol prevents $\mathrm{PM}_{2.5}$-indiced adiposity and onsulin resistance by restraining oxidative stress related ti NF- $\mathrm{B}$ pathway and modulation of gut microbiota in a murine model. Free Radic. Biol. Med. 2019, 141, 393-407. [CrossRef] [PubMed]

45. Cao, K.; Xu, J.; Zou, X.; Li, Y.; Chen, C.; Zheng, A.; Li, H.; Li, H.; Szeto, I.M.Y.; Shi, Y.; et al. Hydroxytyrosol prevents diet-induced metabolic syndrome and attenuates mitochondrial abnormalities in obese mice. Free Radic. Biol. Med. 2014, 67, 396-407. [CrossRef] [PubMed]

46. Stern, J.H.; Rutkowski, J.M.; Scherer, P.E. Adiponectin, Leptin, and Fatty Acids in the Maintenance of Metabolic Homeostasis through Adipose Tissue Crosstalk. Cell Metab. 2016, 23, 770-784. [CrossRef] [PubMed]

47. Flachs, P.; Mohamed-Ali, V.; Horakova, O.; Rossmeisl, M.; Hosseinzadeh-Attar, M.J.; Hensler, M.; Ruzickova, J.; Kopecky, J. Polyunsaturated fatty acids of marine origin induce adiponectin in mice fed a high-fat diet. Diabetologia 2006, 49, 394-397. [CrossRef] [PubMed]

48. Pérez-Matute, P.; Pérez-Echarri, N.; Martínez, J.A.; Marti, A.; Moreno-Aliaga, M.J. Eicosapentaenoic acid actions on adiposity and insulin resistance in control and high-fat-fed rats: Role of apoptosis, adiponectin and tumour necrosis factor- $\alpha$. Br. J. Nutr. 2007, 97, 389-398. [CrossRef]

49. Itoh, M.; Suganami, T.; Satoh, N.; Tanimoto-Koyama, K.; Yuan, X.; Tanaka, M.; Kawano, H.; Yano, T.; Aoe, S.; Takeya, M.; et al. Increased adiponectin secretion by highly purified eicosapentaenoic acid in rodent models of obesity and human obese subjects. Arterioscler. Thromb. Vasc. Biol. 2007, 27, 1918-1925. [CrossRef]

50. Kalupahana, N.S.; Claycombe, K.; Newman, S.J.; Stewart, T.; Siriwardhana, N.; Matthan, N.; Lichtenstein, A.H.; Moustaid-Moussa, N. Eicosapentaenoic Acid Prevents and Reverses Insulin Resistance in High-Fat Diet-Induced Obese Mice via Modulation of Adipose Tissue Inflammation. J. Nutr. 2010, 140, 1915-1922. [CrossRef]

51. Lorente-Cebrian, S.; Bustos, M.; Marti, A.; Martinez, J.A.; Moreno-Aliaga, M.J. Eicosapentaenoic acid stimulates AMP-activated protein kinase and increases visfatin secretion in cultured murine adipocytes. Clin. Sci. 2009, 117, 243-249. [CrossRef]

52. González-Périz, A.; Horrillo, R.; Ferré, N.; Gronert, K.; Dong, B.; Morán-Salvador, E.; Titos, E.; Martínez-Clemente, M.; López-Parra, M.; Arroyo, V.; et al. Obesity-induced insulin resistance and hepatic steatosis are alleviated by $\omega-3$ fatty acids: A role for resolvins and protectins. FASEB J. 2009, 23, 1946-1957. [CrossRef] [PubMed] 
53. Kopecky, J.; Rossmeisl, M.; Flachs, P.; Kuda, O.; Brauner, P.; Jilkova, Z.; Stankova, B.; Tvrzicka, E.; Bryhn, M. n-3 PUFA: Bioavailability and modulation of adipose tissue function. Proc. Nutr. Soc. 2009, 68, 361-369. [CrossRef]

54. Moreno-Aliaga, M.J.; Lorente-Cebrián, S.; Martínez, J.A. Regulation of adipokine secretion by n-3 fatty acids. Proc. Nutr. Soc. 2010, 69, 324-332. [CrossRef] [PubMed]

55. Gray, B.; Steyn, F.; Davies, P.S.W.; Vitetta, L. Omega-3 fatty acids: A review of the effects on adiponectin and leptin and potential implications for obesity management. Eur. J. Clin. Nutr. 2013, 67, 1234-1242. [CrossRef] [PubMed]

56. Giordano, E.; Dávalos, A.; Visioli, F. Chronic hydroxytyrosol feeding modulates glutathione-mediated oxido-reduction pathways in adipose tissue: A nutrigenomic study. Nutr. Metab. Cardiovasc. Dis. 2014, 24, 1144-1150. [CrossRef]

57. Anderson, C.M.; Stahl, A. SLC27 fatty acid transport proteins. Mol. Aspects Med. 2013, 34, 516-528. [CrossRef]

58. Rossi, A.S.; Lombardo, Y.B.; Chicco, A.G. Lipogenic enzyme activities and glucose uptake in fat tissue of dyslipemic, insulin-resistant rats: Effects of fish oil. Nutrition 2010, 26, 209-217. [CrossRef]

59. LeMieux, M.J.; Kalupahana, N.S.; Scoggin, S.; Moustaid-Moussa, N. Eicosapentaenoic Acid Reduces Adipocyte Hypertrophy and Inflammation in Diet-Induced Obese Mice in an Adiposity-Independent Manner. J. Nutr. 2015, 145, 411-417. [CrossRef]

60. Rong, J.X.; Qiu, Y.; Hansen, M.K.; Zhu, L.; Zhang, V.; Xie, M.; Okamoto, Y.; Mattie, M.D.; Higashiyama, H.; Asano, S.; et al. Adipose Mitochondrial Biogenesis Is Suppressed in $d b / d b$ and High-Fat Diet-Fed Mice and Improved by Rosiglitazone. Diabetes 2007, 56, 1751-1760. [CrossRef]

61. de Ferranti, S.; Mozaffarian, D. The perfect storm: Obesity, adipocyte dysfunction, and metabolic consequences. Clin. Chem. 2008, 54, 945-955. [CrossRef]

62. Masschelin, P.M.; Cox, A.R.; Chernis, N.; Hartig, S.M. The Impact of Oxidative Stress on Adipose Tissue Energy Balance. Front. Physiol. 2020, 10, 1-8. [CrossRef] [PubMed]

63. Kusunoki, C.; Yang, L.; Yoshizaki, T.; Nakagawa, F.; Ishikado, A.; Kondo, M.; Morino, K.; Sekine, O.; Ugi, S.; Nishio, Y.; et al. Omega-3 polyunsaturated fatty acid has an anti-oxidant effect via the Nrf-2/HO-1 pathway in 3T3-L1 adipocytes. Biochem. Biophys. Res. Commun. 2013, 430, 225-230. [CrossRef] [PubMed]

64. Tatsumi, Y.; Kato, A.; Sango, K.; Himeno, T.; Kondo, M.; Kato, Y.; Kamiya, H.; Nakamura, J.; Kato, K. Omega-3 polyunsaturated fatty acids exert anti-oxidant effects through the nuclear factor (erythroidderived 2)-related factor 2 pathway in immortalized mouse Schwann cells. J. Diabetes Investig. 2019, 10, 602-612. [CrossRef] [PubMed]

65. Meital, L.T.; Windsor, M.T.; Perissiou, M.; Schulze, K.; Magee, R.; Kuballa, A.; Golledge, J.; Bailey, T.G.; Askew, C.D.; Russell, F.D. Omega-3 fatty acids decrease oxidative stress and inflammation in macrophages from patients with small abdominal aortic aneurysm. Sci. Rep. 2019, 9, 1-11. [CrossRef] [PubMed]

66. Calder, P.C. Omega-3 fatty acids and inflammatory processes: From molecules to man. Biochem. Soc. Trans. 2017, 45, 1105-1115. [CrossRef] [PubMed]

67. Yamada, H.; Umemoto, T.; Kakei, M.; Momomura, S.; Kawakami, M.; Ishikawa, S.; Hara, K. Eicosapentaenoic acid shows anti-inflammatory effect via GPR120 in 3T3-L1 adipocytes and attenuates adipose tissue inflammation in diet-induced obese mice. Nutr. Metab. 2017, 14,1-11. [CrossRef]

68. Kruglikov, I.L.; Scherer, P.E. The Role of Adipocytes and Adipocyte-Like Cells in the Severity of COVID-19 Infections. Obesity 2020, 28, 1187-1190. [CrossRef]

69. Kokeny, G.; Calvier, L.; Legchenko, E.; Chouvarine, P.; Mozes, M.M.; Hansmann, G. PPAR-gama is a gatekeeper for extracellular matrix and vascular cell homeostasis: Beneficial role is $\mathrm{n}$ pulmonary hypertension and renal/cardiac/pulmonary fibrosis. Curr. Opin. Nephol. Hypertens. 2020, 29, 171-179. [CrossRef]

Sample Availability: Samples of the compounds are not available from the authors. 